\section{DEFINING STAGES OF THE INDUSTRY 4.0 ADOPTION VIA INDICATOR SETS}

\author{
Celbert Himang [i] Lanndon Ocampo* (1) \\ JUN-JUN OBISO MIRIAM BONGO \\ Shirley ANN Caballes ${ }^{\circledR}$ Dharyll Prince Abellana \\ Custer DeOcaris ${ }^{(1)}$ ROSEIN ANCHETA, JR.
}

pages: $32-55$

Lanndon Ocampo

Cebu Technological University, Philippines ORCID 0000-0002-5050-7606

*Corresponding author: e-mail: lanndonocampo@gmail.com

Celbert Himang

Cebu Technological University, Philippines ORCID 0000-0002-5663-3304

Jun-Jun Obiso

Cebu Technological University, Philippines ORCID 0000-0001-5726-7812

\section{A B S TR A C T}

As Industry 4.0 offers significant productivity improvements, its relevance has grown across various organisations. While it captures the attention of both the industry and the academia, very few efforts have been made to streamline useful indicators across stages of its implementation. Such work facilitates the development of strategies that are appropriate for a specific stage of implementation; therefore, it would be significant to a variety of stakeholders. As a result, this paper aims to establish an indicator system for adopting Industry 4.0 within the context of the three stages of the innovation adoption: (i) pre-adoption, (ii) adoption, and (iii) post-adoption. First, a comprehensive review was performed with a search expanding into the literature on innovation and technology adoption. Second, the resulting indicators were filtered for relevance, redundancy, description, and thorough focus discussions. Finally, they were categorised by their stage of adoption. From 469 innovation adoption indicators found in the literature, this work identified a total of 62 indicators relevant for the Industry 4.0 adoption, in which 11, 14, and 37 of them comprised the three stages, respectively. Case studies from two manufacturing firms in the Philippines were reported to demonstrate the applicability of the proposed indicator system. This work pioneers the establishment of an indicator system for the Industry 4.0 adoption and the classification of such indicators into three stages - pre-adoption, adoption, and post-adoption which would serve as a framework for decision-makers, practitioners, and stakeholders in planning, strategy development, resource allocation, and performance evaluation of the Industry 4.0 adoption.

KEY WORDS

Industry 4.0, indicators, stages of adoption, management of Industry 4.0
Miriam Bongo

Cebu Technological University, Philippines ORCID 0000-0002-9383-3399

Shirley Ann Caballes

Cebu Technological University, Philippines ORCID 0000-0001-7261-4755

Dharyll Prince Abellana

Cebu Technological University, Philippines ORCID 0000-0002-2397-556X

Custer Deocaris

Commission on Higher Education, Philippines ORCID 0000-0003-3530-1228

Rosein Ancheta, Jr. Cebu Technological University, Philippines

\title{
INTRODUCTION
}

In various organisations, the quick shift towards digital transformation has been primarily modifying business models, production processes, and corporate governance methods. As such, the rapid stride of technological advancement necessitates the decision to adopt innovations. Consequently, companies that have more innovation capabilities are also more able to recognise early the extent of the influence by the digital transformation on their business models as well as the contribution they can get from the information derived from their initiatives (OECD, 2005).

Citation: Himang, C., Ocampo, L., Obiso, J.-J., Bongo, M., Caballes, S. A., Abellana, D. P., Deocaris, C., \& Ancheta, R. Jr. (2020). Defining stages of Industry 4.0 adoption via indicator sets. Engineering Management in Production and Services, 12(2), 32-55. doi: 10.2478/emj-2020-0010 
The potentials offered by the increasing application of digitisation are reshaping the competitive dispositions of organisations, their customer and employee interrelation, and market positioning (CasteloBranco et al., 2019). Hence, the capability of firms to grasp the concepts and applications behind digitisation has become crucial in gaining competitive advantage (Bleicher \& Stanley, 2016).

The area of digital transformation contains a dimension that has been capturing the interest of academics and practitioners concerning the prospects and the effect of applying digitisation to organisations, which is commonly termed Industry 4.0 (I4.0). Rapid changes brought by I4.0 modified how an organisation operates. Primarily, the principle of I4.0 is the interconnectivity of digital technologies, devices, and processes, which enables the operation of autonomous manufacturing models, able to perform in a decentralised decision setting with minimal human interference, and capable of connectedly working together along the stages of the production process and across several stages of the supply chain (Castelo-Branco et al., 2019).

Innovations and changes in corporate environments significantly affect a firm's performance and sustainability. Furthermore, firms need to create appropriate strategies to aid their preparation for future emerging industrial developments, for instance, I4.0. This is especially relevant when the path towards a completely digital manufacturing enterprise is ambiguous (Lee et al., 2013). In fact, the current I4.0 trend has not yet been recognised by several industry leaders. Some do acknowledge this industry trend, however, they are generally unaware of the initiatives for making their organisations prepared for the I4.0 implementation (Rajnai \& Kocsis, 2018). Nevertheless, converting a firm into a completely digital enterprise requires the alteration of organisation's strategies, which is an essential choice to make aiming for the success and sustainability of competitive advantage in the digital transformation process essential for I4.0 (Vogel-Heuser \& Hess, 2016). To this end, establishing an indicator system proves to be relevant for appropriately steering an organisation's strategic direction and evaluating ideas and concepts further, especially across the stages of the process of innovation.

Various studies on the topic of I4.0 focused on such issues as streamlining the opportunities or challenges of I4.0 (Kamble et al., 2018; Glass et al., 2018; Müller et al., 2018), I4.0 development indicators (Alekseev et al., 2018), antecedents to the use of I4.0
(Müller, 2019), critical success factors (de Sousa Jabbour et al., 2018), and, more abundantly, on the aspect of technicality and key technologies, such as cyberphysical systems (CPS) (Lee et al., 2015; Alguliyev et al., 2018), the Internet of Things (IoT) (Hsu and Lin, 2016), cloud computing (Priyadarshinee et al., 2017; Hassan, 2017; Siderska \& Mubarok, 2018), and smart manufacturing (Tao et al., 2018). However, despite current studies, the focus is rarely placed on I4.0 indicators, even though the topic requires further analysis.

For most companies, the establishment of indicators is deemed crucial for better management and control of emerging concepts and ideas regarding innovation. Furthermore, indicators are significant for an appropriate and efficient allocation of resources, and assessment of performance at a specific innovation stage (Dewangan \& Godse, 2014). In policymaking initiatives, having an appropriate set of indicators can aid an organisation's status and level of the I4.0 implementation, further recognising the relevance and suitability of innovation activities completed to realise the full implementation of I4.0. Given the necessity to improve and develop an indicator system for I4.0, the present study attempts to provide a set of indicators behind the performance management in the implementation of I4.0 tailored according to the stages of innovation (OECD, 2005; Birchall et al., 2011).

Consequently, it is essential to consider arguments used by various innovation scholars over the past two decades, such as Rogers (1995), Hameed et al. (2012), and Caiazza and Volpe (2016), indicating that any innovation adoption occurs in stages. For instance, Hameed et al. (2012) argued that the innovation process could be summarised in three stages: pre-adoption, adoption-decision, and post-adoption. Thus, the management of the I4.0 implementation should follow a stage-based approach since different concerns prevail at different stages (Hameed et al., 2012). The understanding of the issues particular to different stages enables firms to suitably craft programmes and initiatives for gaining competitive advantage, making resource allocation decisions, and long-term planning. The classification of innovation maturity into stages has demonstrated its usefulness in business, as demonstrated by the current literature. For example, Solis (2016) classified digital transformation maturity into six levels: (1) business as usual, (2) test and learn, (3) systemise, (4) adapt or die, (5) transformed and transforming, and (6) innovate or die. Habicht et al. (2012) defined the stages of open 
innovation as (1) staying closed, (2) defined open innovation, (3) managed open innovation, and (4) aligned open innovation. Moreover, Ham et al. (2015) categorised the maturity of open innovation for the government into four stages: (1) semi-opened, (2) focused-opened, (3) balanced-opened, and (4) fully opened.

Unfortunately, the I4.0 implementation has not been viewed in terms of its distinct stages of adoption despite being under the umbrella of the general innovation domain. In the current literature, the I4.0 implementation has been short-sighted and fragmented as it is deliberately embedded in existing management frameworks. Such approaches diverge from the conventional innovation theory established by Rogers (1995). These approaches may fail to establish a holistic method embedded in the innovation process, which may result in haphazard implementation, waste of resources, and a myopic view of I4.0.

Thus, this work attempts to address two critical gaps in the literature: (1) treating I4.0 as an innovation process, which is described in stages, and (2) developing indicator sets for each stage of innovation. The objective of this work is to reveal indicators specific for stages of the I4.0 adoption, which would guide decision-makers in strategy development and evaluation as well as performance evaluation. Evanschitzky et al. (2012) supported the notion that indicators played significant roles in efficient resource allocation and performance evaluation. These indicators were characterised by measurable parameters that could provide valuable information (Dziallas \& Blind, 2018) about the adoption capabilities and necessities of firms at each stage. Thus, in the process of I4.0 adoption, the need to identify the operational adoption indicators for each stage becomes essential. Case studies from two manufacturing firms operating in the Philippines are reported in this work to demonstrate the applicability of the proposed indicator system across all stages of innovation. A generic methodological framework is offered, but the specific mathematical toolbox that encapsulates the entire framework is reserved for future work. Without compromising generality, the used approach was derived from the outline of $\mathrm{Xu}$ (2006) on the linguistic arithmetic averaging operator.

The paper has six sections. This section is followed by Section 2, which rationalises the stages of innovation adoption. Section 3 discusses the methods for the selection of different adoption indicators. Adoption indicators for each stage of adoption are identified in Section 4. Section 5 elaborates on the applications of the proposed indicator sets by using case studies conducted in manufacturing firms operating in the Philippines. Finally, Section 6 presents managerial implications and concluding remarks.

\section{STAGES OF THE INNOVATION ADOPTION}

Schumpeter (1934) first defined innovation as a combination of new or existing knowledge, resources, equipment, and other factors. In the Manual on the Measurement of Scientific and Technological Activities, this definition was adopted by OECD (2005) as the implementation of a new or significantly improved product, process, or service. Crossan and Apaydin (2010) extended the definition of innovation as "production or adoption, assimilation, and exploitation of a value-added novelty in economic and social spheres: renewal and engagement of products, services and markets; development of new methods of production; and the establishment of new management systems." The above description was simplified by Edison et al. (2013), emphasising two essential concepts: first, there must be an invention or discovery of a new idea, and second, there must be commercialisation or successful exploitation through commercialisation of such discovery. The latter description of innovation emphasises the commercialisation, which offers a better picture of I4.0 as innovation. For a more elaborate discussion on the commercialisation component of innovation, see the works of Slater and Mohr (2006), Datta et al. (2013), Datta et al. (2015), and Egorova et al. (2017). For brevity, and as the topic falls outside the scope of this work, the emphasis on commercialisation as a crucial point of innovation is not presented here.

Hermann et al. (2016) considered Industry 4.0 as a convergence between industrial production and information and communication technologies (ICT), which is comparable to technical innovation (Oesterrich \& Teuteberg, 2016) and technological innovation (Kagermann et al., 2013). Kamble et al. (2018) stressed that the ICT part of I4.0 consisted of the cyber-physical system (CPS), cloud computing, and the Internet of Things. This position justifies the need to untangle the I4.0 adoption from the context of innovation adoption as the infrastructure of I4.0 is mostly ICT-based. Thus, since I4.0 occurs within the innovation context, it is apparent that any work on I4.0 must be anchored in the foundation of innovation studies. In the light of the innovation domain, 
van Oorschot et al. (2018) used both bibliometric coupling and co-citation analysis to map and synthesise fragmented empirical studies on innovation, which revealed the theory of Diffusion of Innovation (DOI) by Rogers as the cornerstone of innovation adoption research.

As described by Rogers (1995), innovation adoption is a process that occurs mainly from awareness or knowledge, attitude formation to persuasion to a decision to adopt or reject, then followed by implementation. Moreover, since the innovation process is usually complicated (Dodgson and Hinze, 2000), it is apparent to embrace the concept of indicators to understand innovation adoption. As claimed by Cavdar and Aydin (2015), indicators are crucial for information about things that are difficult to measure. Caiazza and Volpe (2016) asserted that indicators are indispensable to management and control of the plethora of innovative ideas and concepts. Gault (2018) highlighted that indicators could be used for monitoring and evaluation of implemented innovation policies. Likewise, Evanschitzky et al. (2012) inferred that for policy-making practices, it is significant to have accurate indicators to evaluate the proposed innovation and the impact of such innovation. On the other hand, Dziallas and Blind (2018) reported that innovation process indicators are less frequently investigated. Thus, it is crucial to unfold the complexities of the I4.0 adoption by espousing the concept of indicator sets. However, despite the importance, the identification of I4.0 indicators has not been explored in the current literature.

Relevant literature on the innovation adoption indicators, which is not specifically within the context of I4.0, reported different frameworks and phases of the innovation process. Hart et al. (2003) held that the early stages of the innovation process required different indicators in comparison with a later stage. Their notion was derived from their investigation of the new product development (NPD) process with stages that included the idea generation, concept development, building the business case, product development, market testing, and market launch. Also, in terms of a lifecycle-oriented approach (Suomala, 2004) to innovation, Dewangan and Godse (2014) argued that each phase of innovation lifecycle had its unique activities and outputs, amenable to measurement and benchmarking. Evanschitzky et al. (2012) established selection criteria for efficient resource allocation and performance evaluation at each phase of the innovation process. Lombardi et al. (2013) introduced a novel framework for classifying smart city components and performance indicators. They clustered the indicators as smart governance, smart human capital; smart environment; smart living, and smart economy. Dziallas and Blind (2018) introduced process innovation indicators and factors in the framework of the stage-gate system introduced by Cooper (1990). Similarly, Miremadi et al. (2018) proposed an energy innovation indicator framework that focused on the energy innovation process, covering the entire innovation chain and incorporating indicators into the specific innovation stages. However, these studies on the concept of innovation indicators did not use the model of the innovation process by Rogers categorically as the cornerstone of innovation research, as reported by van Oorschot (2018). This model was summarised from innovation studies by Damanpour and Schneider (2006) and Hameed et al. (2012) as a pre-adoption stage, adoption-decision stage, and post-adoption stage.

The pre-adoption or initiation stage involves activities similar to need or problem recognition, information search on the innovation's existence, forming an attitude towards the innovation, and proposing innovation for adoption (Rogers, 1995; Hinnant \& O'Looney, 2003). Hence, this stage is considered as the preparatory stage of adoption. The adoption-decision stage, on the other hand, manifests acceptance or rejection of the innovative idea based on the evaluation of human and material resources and the assessment and future resource allocation if the innovative idea is accepted (Hameed et al., 2012). It is the stage, wherein the adopters have entirely accepted or rejected the innovation for actual implementation. The post-adoption stage, also known as the implementation stage, encompasses possession, validation, acceptance, and sustained real use of the innovation (Hameed et al., 2012).

Moreover, Zhu et al. (2006), grounded in the DOI theory and the Technology-Organisation-Environment (TOE) framework, established four innovation characteristics (relative advantage, compatibility, costs, and security concern) and four contextual factors (technology competence, organisation size, competitive pressure, and partner readiness) as determinants of the post-adoption usage. It is the stage, wherein the adopters have applied the innovation in the system with full acceptance of the benefits and risks brought about by such adoption. With the four characteristics of innovation, I4.0 can be considered an innovation. First, it exhibits a relative advantage, as found by Arnold et al. (2018). Second, through decentralisation, I4.0 becomes easily com- 
patible with different organisations, as argued by Shamim et al. (2016). Third, I4.0 is highly related to different types of costs (e.g., production or quality) in a positive sense (Rojko, 2017). Finally, I4.0 has been associated with several security concerns, particularly cyber and information security (Wegner et al., 2017). Having established that I4.0 is an innovation, it follows that the three stages of the adoption of innovation, as prescribed by Rogers (1995), can also be used to categorise the stages of its adoption.

\section{INDICATORS OF THE INDUSTRY 4.0 IMPLEMENTATION}

This section illustrates how to establish the final list of indicators by way of a comprehensive review of related literature and demonstrates the applicability of the list by conducting relevant case studies. Specifically, this process begins with a keyword search in four core databases, followed by a collection of articles and content analysis. Then, indicators are selected according to the context relevance and redundancy.

\subsection{ARTICle SElection PROCESS AND CON- TENT ANALYSIS}

A keyword search was performed in four core databases to gather relevant articles in the literature, which potentially discusses the indicator system for I4.0. As the development of I4.0 is yet an emerging domain and is still at its early stages (Issa et al., 2018), the search was expanded from I4.0-specific applications to the general technology and innovation adoption. The primary keywords used were: "digital transformation", "industry 4.0", "industry 4.0 adoption", "innovation", "innovation adoption", "technology", "technology adoption"; together with supplementary keywords such as "indicators" and "predictors" The study used the following databases: Elsevier's ScienceDirect and Scopus, Taylor \& Francis' www.tandfonline.com, and Springer's SpringerLink. To reach a comprehensive coverage of publications related to the indicators of the I4.0 adoption, journal articles, and conference proceedings were also obtained from these databases. In the following step, a content analysis was performed to extract prospective indicators on identified articles. Articles that do not ultimately provide related indicators were excluded.

\subsection{SELECTION OF INDICATORS}

A comprehensive list of indicators was generated from a variety of innovation studies, having numerical metrics as part of their methodology. However, in the context of I4.0, no present study was able to develop a set of indicators to assess the I4.0 implementation at different stages. Thus, a significant challenge was to select an appropriate set of indicators from the general system of innovation and technology adoption. Addressing the challenge, several criteria were used for the selection and construction of indicators. Four criteria were used in the screening process to select appropriate indicators. Miremadi, Saboohi and Jacobsson (2018) developed general criteria for the selection of indicators in the context of innovation systems. They used this set of criteria as it covered approximately all factors in the relevant literature (Miremadi, Saboohi \& Jacobsson, 2018). To measure the I4.0 implementation, indicators must be understandable, available, relevant, and measurable. First, an indicator is considered understandable if it is straightforward, simple, and provides ease of understanding. Second, an indicator is available if data and information are accessible. Availability ensures that the value of a specific indicator is obtainable from a company's information system. Third, indicators are deemed relevant if they satisfy the goal of assessing the level of the I4.0 implementation and if they point to the characteristics or nature of activities per stage. Fourth, indicators must be measurable following an existing scientific measurement approach (e.g., surveys).

An initial list of indicators was generated from a literature review on a variety of technology and innovation adoption applications, as shown in Table 1. In this work, an indicator was defined as a source of information, from which problems could be detected in the application of innovation (Borras \& Edquist, 2013). A total of 469 indicators were collected. At the outset, these indicators contained literal redundancies of terminologies in different sources. Consequently, such redundant indicators were excluded, and this process yielded 90 candidate indicators. Afterwards, an appropriate description of each indicator was provided, indicating primary sources, from which they were extracted. In cases of insufficiency, supplementary or secondary sources (i.e., related journal articles and scholarly books) were used. From the initial list of indicators with descriptions, specific terms were found to be synonymous. Indicators implying a synonymous meaning were treated 
as redundant, thus, excluded. Focus meetings were then conducted to qualify a final list of indicators.

Descriptions of each indicator were carefully assessed. Each indicator was then assessed using the four criteria, focusing on its understandability, availability, relevance, and measurability. Subsequently, following the process of a thorough assessment, indicators that did not meet the four criteria were rejected. This process generated a final list of 62 indicators of the I4.0 adoption. An initial list was then categorised according to stages of adoption from initiation, adoption-decision, and implementation (i.e., pre-adoption, adoption, and post-adoption, respectively) (Hameed et al., 2012). These stages were an essential determinant to reflect the entire innovation process and to control the applicability of each indicator (Table 2).

Table 1 presents the number of extracted indicators, their application, and sources to provide an overview of the initial listing of I4.0 indicators used in this paper. The first column indicates authors from whom candidate I4.0 indicators were extracted. Papers listed under the label "reference indicators" indicate sources used to collate respective innovation or I4.0 indicators. Hence, "reference indicators" are the sources of performed compilation. For instance, to explore and discuss I4.0 technologies, $\mathrm{Lu}$ (2017) collated I4.0 indicators from Jazdi (2014), Stock and Seliger (2016) and Wang et al. (2016), among others. Moreover, the second column comprises the field of

Tab. 1. Numbers of indicators generated from literature with their corresponding application

\begin{tabular}{|c|c|c|}
\hline AUTHOR(s) & APPLICATION & $\begin{array}{c}\text { No. } \\
\text { OF GENERATED } \\
\text { INDICATORS }\end{array}$ \\
\hline Chor et al. (2014) & Contextual level-based innovation adoption & 116 \\
\hline Danquah (2018) & Technology adoption and utilisation & 1 \\
\hline Ezzi and Jarboui (2016), Yigitcanlar et al. (2017) & $\begin{array}{l}\text { Financial, social, and environmental effects of innovation } \\
\text { strategy }\end{array}$ & 2 \\
\hline $\begin{array}{l}\text { Lu (2017) } \\
\text { Reference indicators: } \\
\text { Jazdi (2014), Stock and Seliger (2016), } \\
\text { Wang et al. (2016), Gorecky et al. (2014), Her- } \\
\text { mann et al. (2016), Kolberg and Zühlke (2015) }\end{array}$ & Industry 4.0 technologies & 7 \\
\hline Lee et al. (2015) & Industry 4.0 technologies (CPS) & 1 \\
\hline Tao et al. (2018) & Industry 4.0 technologies (smart manufacturing) & 1 \\
\hline $\begin{array}{l}\text { Hameed et al. (2012) } \\
\text { Reference indicators: Gopalakrishnan and } \\
\text { Damanpour (1997), Rogers (1995), } \\
\text { Meyer and Goes (1988) }\end{array}$ & IT innovation adoption & 124 \\
\hline Jeyaraj et al. (2006), Pilke (2004) & Individual and organisational-based IT innovation adoption & 94 \\
\hline Alguliyev et al. (2018) & Industry 4.0 technologies (CPS) & 14 \\
\hline Attaran (2017) & Industry 4.0 technologies (additive manufacturing) & 5 \\
\hline Hassan (2017) & Industry 4.0 technologies (cloud computing adoption) & 3 \\
\hline Hsu and Lin (2016) & Industry 4.0 technologies (adoption of the Internet of Things) & 6 \\
\hline Letia and Kilyen (2018) & Industry 4.0 technologies (CPS) & 9 \\
\hline Lopez and Rubio (2018) & $\begin{array}{l}\text { Industry } 4.0 \text { technologies (integration of CPS and cloud } \\
\text { computing) }\end{array}$ & 2 \\
\hline Molina and Jacob (2017) & Industry 4.0 technologies (CPS) & 1 \\
\hline Monostori et al. (2016) & Industry 4.0 technologies (CPS) & 4 \\
\hline Priyadarshinee et al. (2017) & Industry 4.0 technologies (cloud computing adoption) & 50 \\
\hline Salleh et al. (2017) & Software functionality service & 1 \\
\hline Sharma et al. (2016) & Industry 4.0 technologies (cloud computing adoption) & 6 \\
\hline Sung (2018) & Industry 4.0 levers & 17 \\
\hline Terziyan et al. (2018) & Industry 4.0 technologies (artificial intelligence) & 5 \\
\hline & TOTAL & 469 \\
\hline
\end{tabular}


application used by a corresponding author (e.g., Chor et al. (2014), Lu (2017), and Hameed et al. (2017)) to demonstrate roles of indicators. The third column displays the number of indicators extracted from corresponding works to comprise the initial list of I4.0 indicators in this paper. The information displayed in Column 3 (Table 1) demonstrates that most of the extracted indicators came from the general innovation literature. For example, 116 indicators were extracted from Chor et al. (2014). The result stems from the attribution of I4.0 to innovations in the current literature, as pointed out by Morrar et al. (2017), Liao (2017), Brettel et al. (2014), and AlmadaLobo (2016), among others. Based on such claims in the literature, placing general innovation indicators in the context of I4.0 is validated by its innovation status.

\subsection{Application OF CASE STUdies}

To illustrate the use of the developed indicator system to assess the I4.0 implementation, case studies involving two manufacturing firms in the Philippines were conducted. The developed indicator set for each stage of the I4.0 adoption intended to assess the degree, to which a firm was positioned, given its current I4.0 implementation. By using the indicator sets, this work offered a general methodological approach, which attempted to generate the value indicating the performance or maturity of the firm at any given stage. The performance or maturity value, now denoted as a general index, provides a snapshot of the performance of the firm at an I4.0 adoption stage at a given time. Note that the quality of this snapshot is highly dependent on the completeness and quality of the information used in the evaluation process, and the level of information granularity of a specific applied methodology. The methodological framework starts with the assignment of weights for the indicators of a given stage. Weight assignment could be carried out using multiple criteria decision-making methods (e.g., analytic hierarchy process, best-worst method, simple average weighting), expert opinion, Delphi method, group decision-making techniques, etc. Once the appropriate I4.0 adoption stage is determined for the firm, the performance or maturity of the firm is assessed against each indicator of the stage, using a specified evaluation scale. The results of the second process are the performance values of the firm for all indicators. Then, using a specified aggregation technique, these performance values are aggregated homogeneously. The aggregation process provides a general dimensionless index that describes the overall performance of the firm at a given stage. Although this work offers the general methodological framework, the specific methodology that embodies the framework is left at the discretion of the firm or its analyst. A detailed procedure in assessing indicators under each I4.0 adoption stage is presented as follows. Note that the proposed procedure is recommendatory, not absolute. A thorough analysis of the most appropriate methodology that maximises the quality of information used in the evaluation process is out of the scope of this work. The following procedure is presented to demonstrate the use of the proposed indicator system in a real-life application:

Step 1: Attain the performance of the indicator. Industry experts firstly identify the current stage of adoption (i.e., pre-adoption, adoption, and postadoption) and further elicit their judgment on each indicator's performance with respect to the perceived adoption stage, using linguistic scales "very poor", "poor", "fair", "good", and "very good", whichever is applicable.

Step 2: Translate the performance of the indicator into a numerical value, according to $\mathrm{Xu}$ (2006). For a given linguistic set, $\mathrm{S}$, a corresponding $s_{\alpha}$, the numerical value is attained for each indicator as in (1),

$$
S=\left\{s_{\alpha} \mid \alpha=-t, \ldots,-1,0,1, \ldots, t\right\}
$$

that is (2),

$$
\begin{gathered}
S=\left\{s_{-2}=\text { very poor, } s_{-1}=\text { poor }, s_{0}=\right.\text { fair, } \\
\left.s_{1}=\text { good, } s_{2}=\text { very good }\right\}
\end{gathered}
$$

correspondingly, these indicator indices will be used to obtain the overall performance of firms at a particular I4.0 adoption stage.

Step 3: Define the overall performance of a firm as regards the I 4.0 adoption. The indices of previously generated indicators are then aggregated as shown in (3) to arrive at a general index on the performance of firms as regards the I4.0 implementation,

$$
\begin{gathered}
L W A A_{\omega}\left(s_{\alpha 1}, s_{\alpha 2}, \ldots, s_{\alpha n}\right)=\omega_{1} s_{\alpha 1} \oplus \omega_{2} s_{\alpha 2} \oplus \ldots \\
\ldots \oplus \omega_{n} s_{\alpha n}=s_{\bar{\alpha}}
\end{gathered}
$$

Where

$\bar{\alpha}=\sum_{j=1}^{n} \omega_{j} \alpha_{j}, \omega=\left(\omega_{1}, \omega_{2}, \ldots, \omega_{n}\right)^{T}$

is the weight vector of the $s_{\alpha_{j}}(j=1,2, \ldots, n)$ and $\omega_{j} \in[0,1], \sum_{j=1}^{n} \omega_{j}=1, s_{\alpha_{j}} \in \bar{S}$, then LWAA is referred to as the linguistic weighted arithmetic averaging (LWAA) operator. In the case of this paper, the weight vector of the $s_{\alpha_{j}}$ is assumed to be equal, thus, the average of $s_{\alpha_{j}}$ is given by (4),

$$
L W A A_{\omega}\left(s_{\alpha 1}, s_{\alpha 2}, \ldots, s_{\alpha n}\right)=\frac{\sum_{j=1}^{n} s_{\alpha 1}, s_{\alpha 2}, \ldots, s_{\alpha n}}{n}
$$




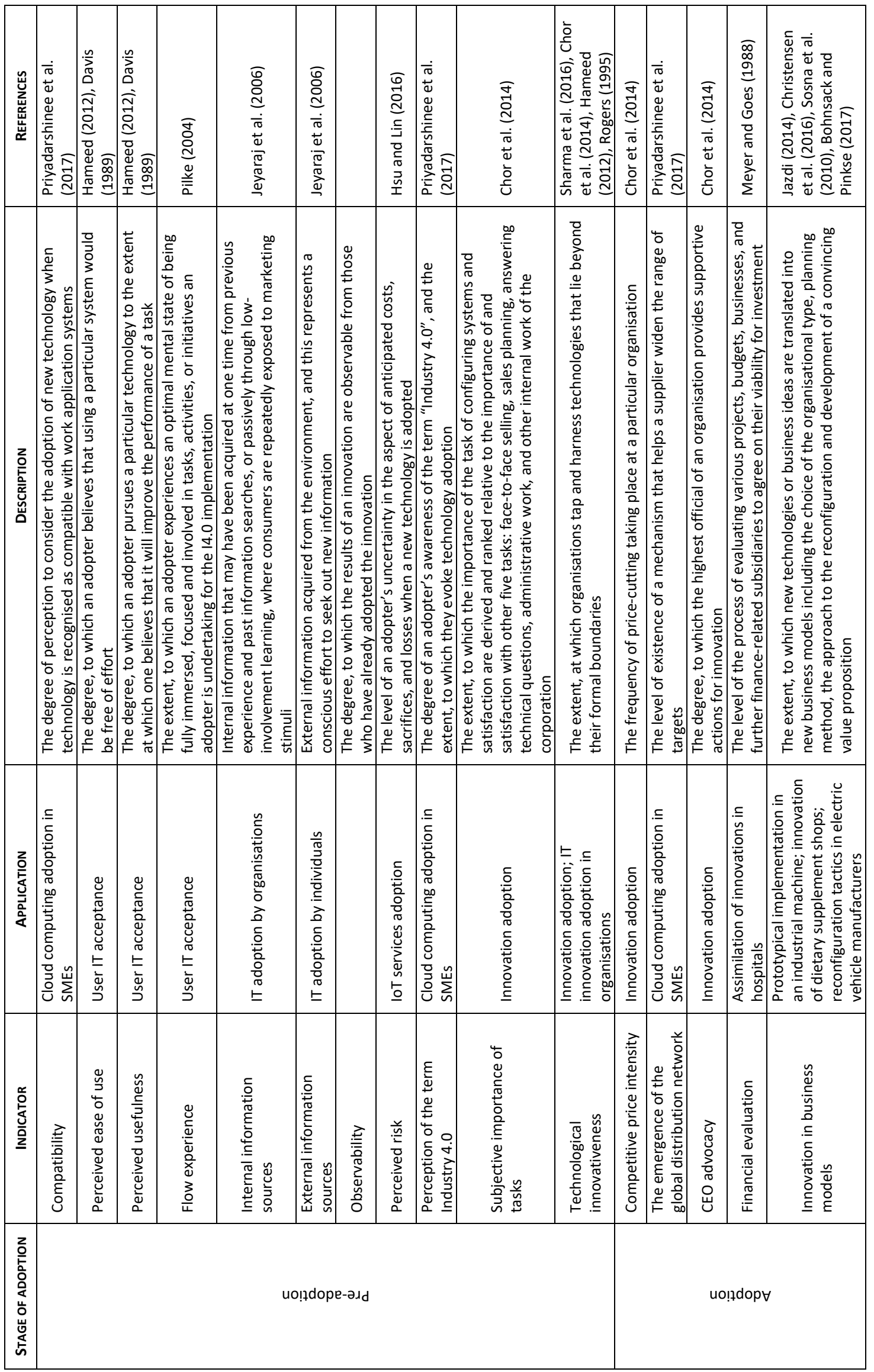




\begin{tabular}{|c|c|c|c|c|c|c|c|c|c|c|c|c|c|}
\hline 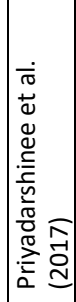 & 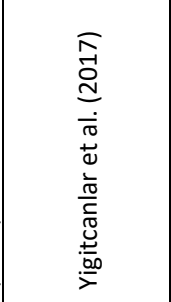 & 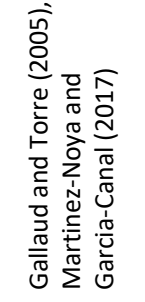 & 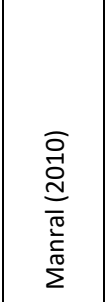 & 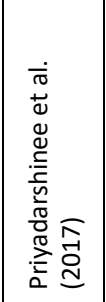 & 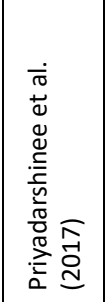 & 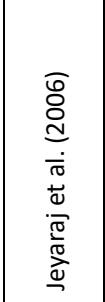 & 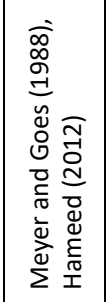 & 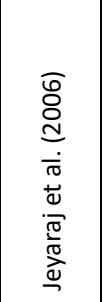 & 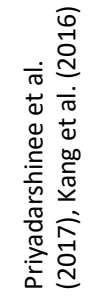 & 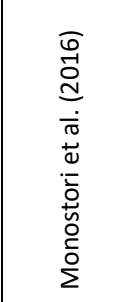 & 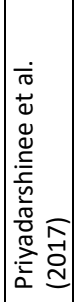 & 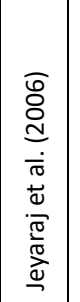 & 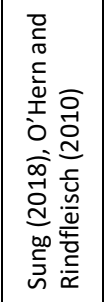 \\
\hline 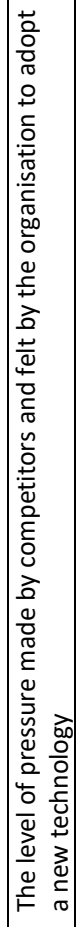 & 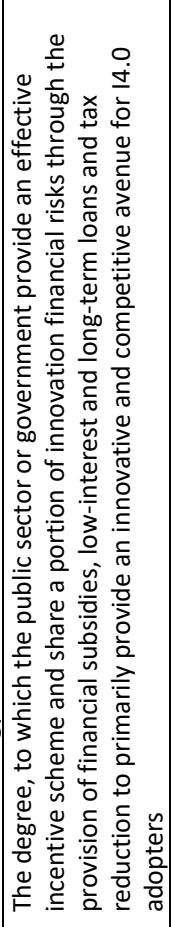 & 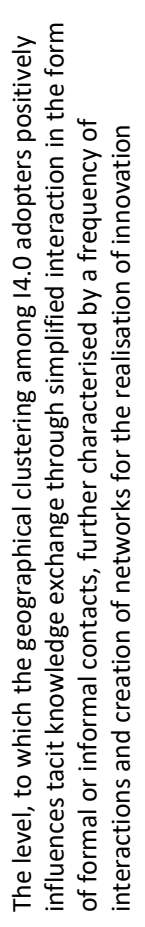 & 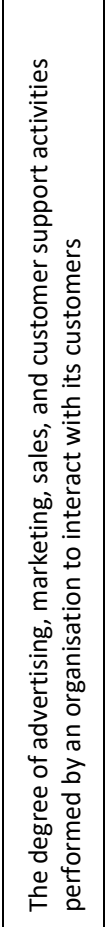 & 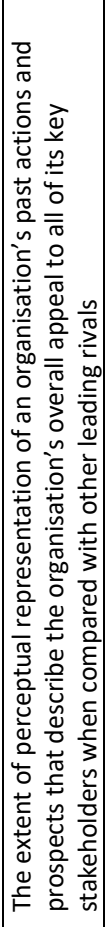 & 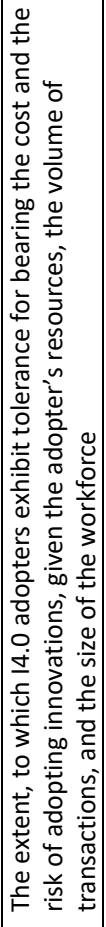 & 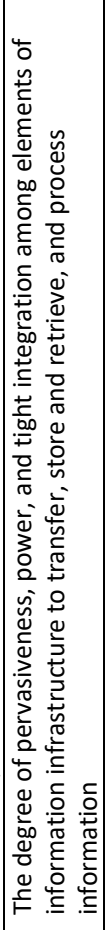 & 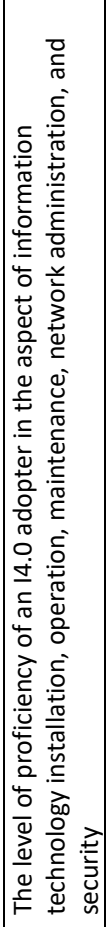 & 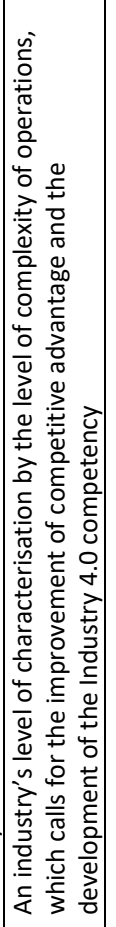 & 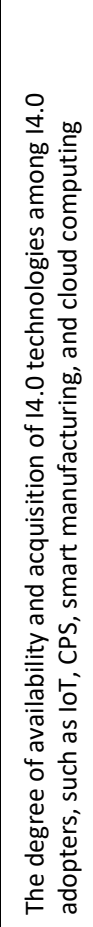 & 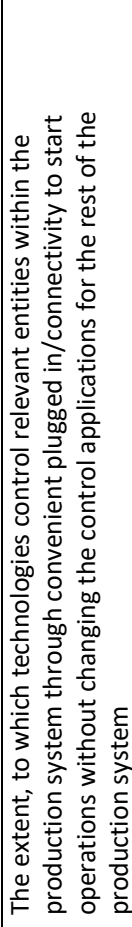 & 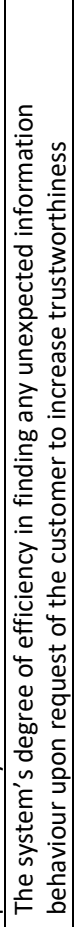 & 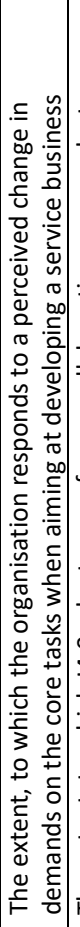 & 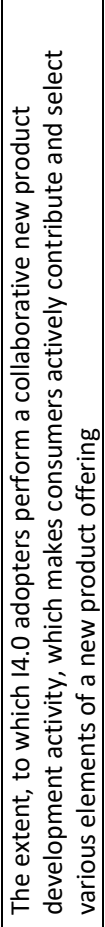 \\
\hline 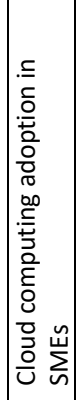 & 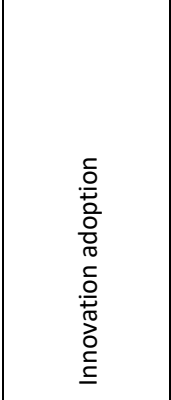 & 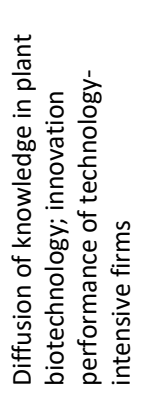 & 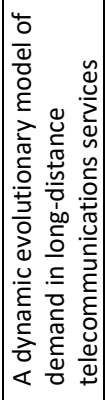 & 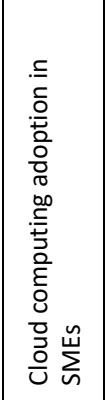 & 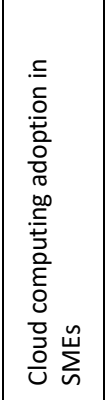 & 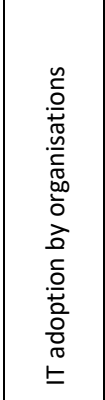 & 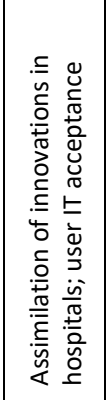 & 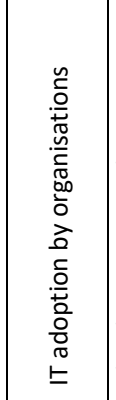 & 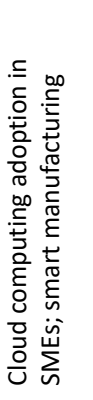 & 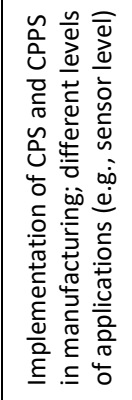 & 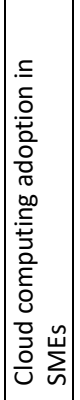 & 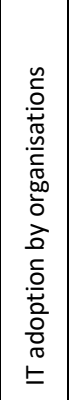 & 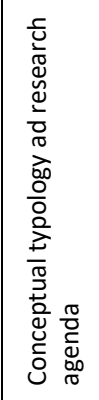 \\
\hline 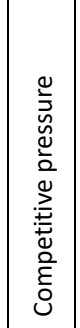 & 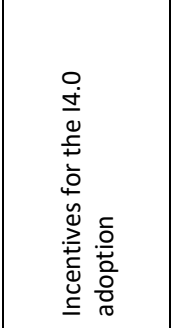 & 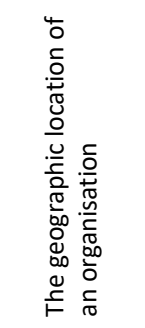 & 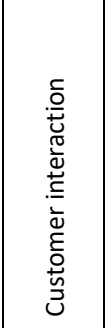 & 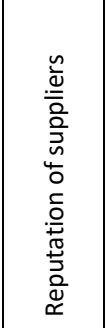 & 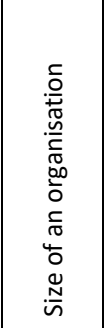 & 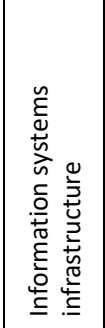 & 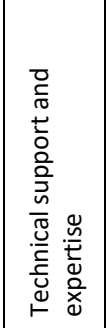 & 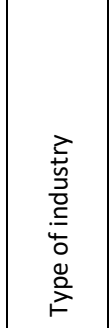 & 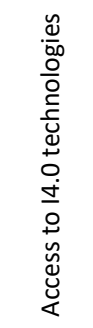 & 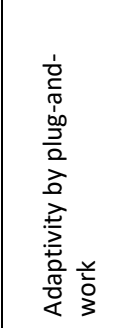 & 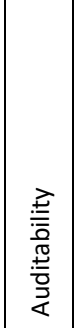 & 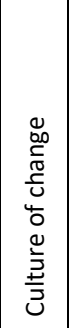 & 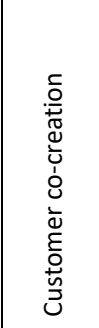 \\
\hline
\end{tabular}




\begin{tabular}{|c|c|c|c|c|c|c|c|c|c|c|c|c|c|c|c|c|}
\hline 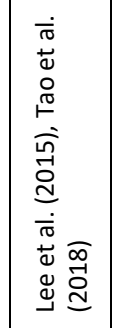 & 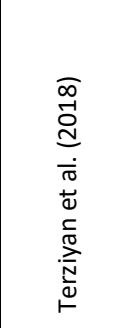 & 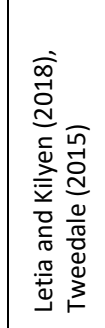 & 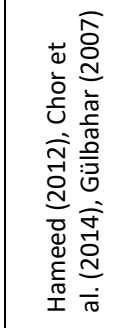 & 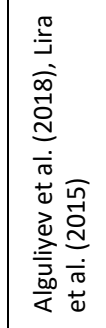 & 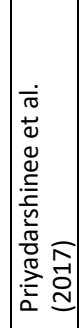 & 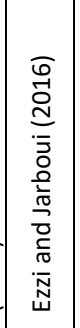 & 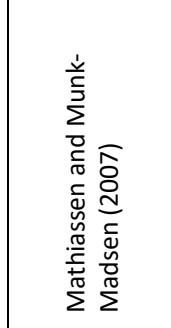 & 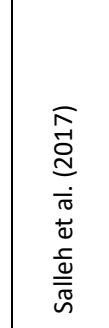 & 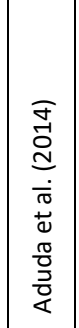 & 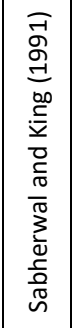 & 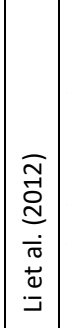 & 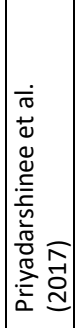 & 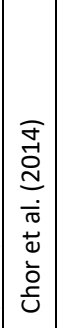 & 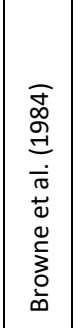 & 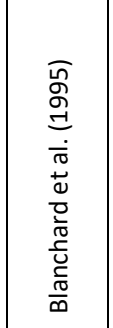 & 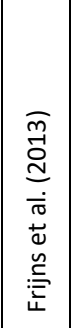 \\
\hline 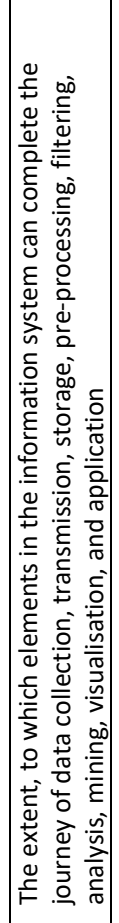 & 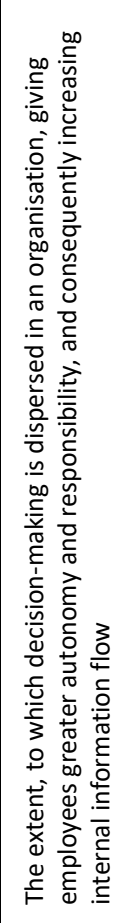 & 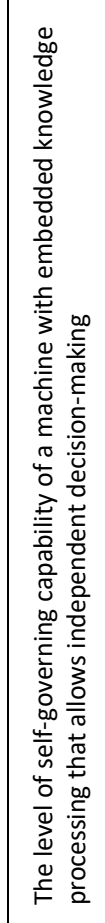 & 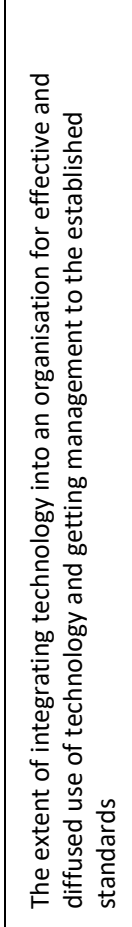 & 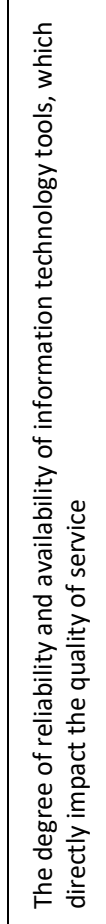 & 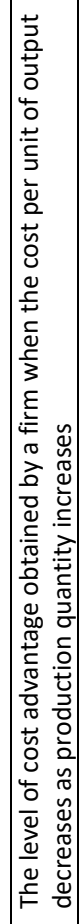 & 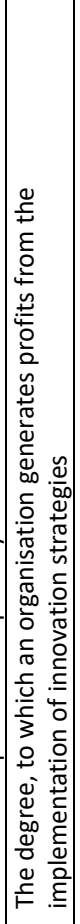 & 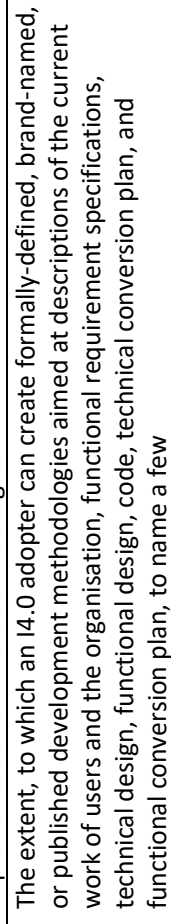 & 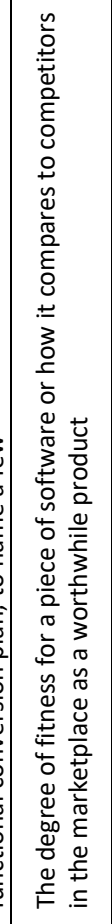 & 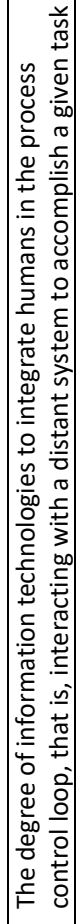 & 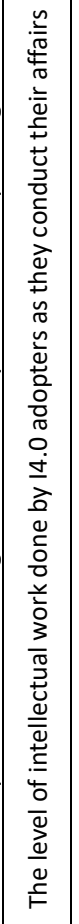 & 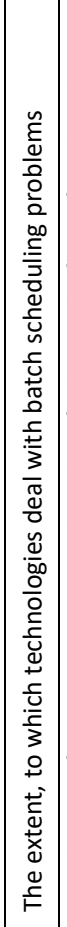 & 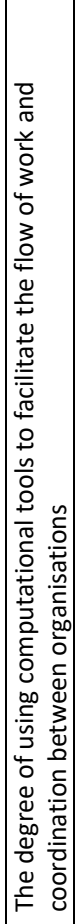 & 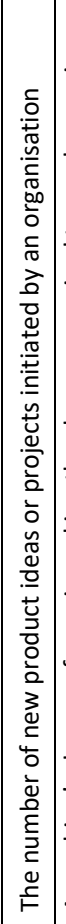 & 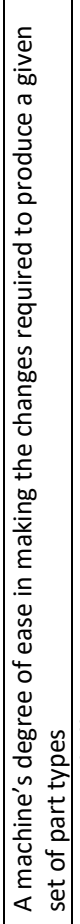 & 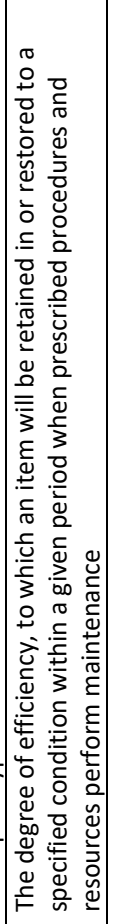 & 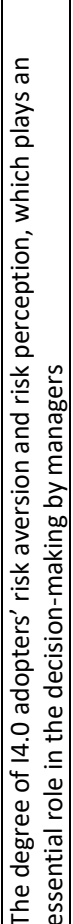 \\
\hline 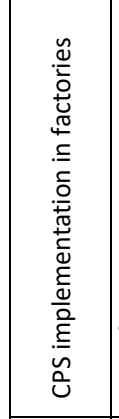 & 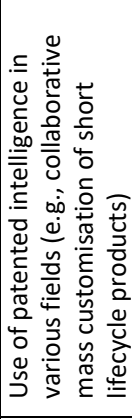 & 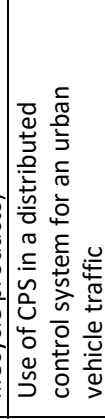 & 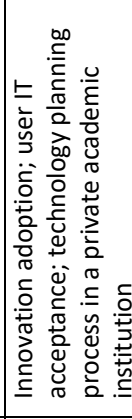 & 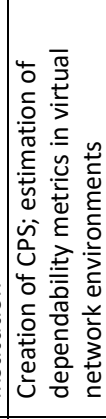 & 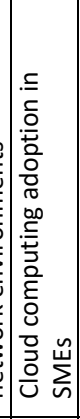 & 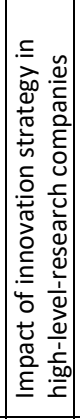 & 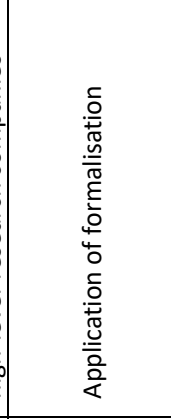 & 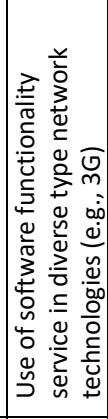 & 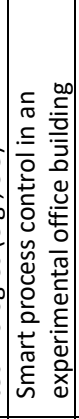 & 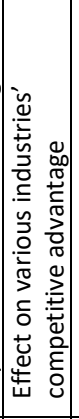 & 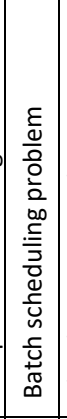 & 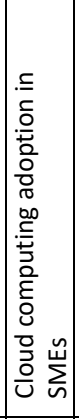 & 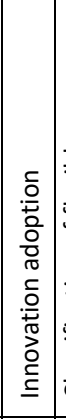 & 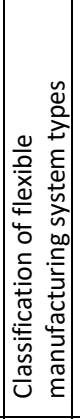 & & 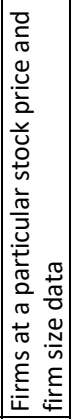 \\
\hline 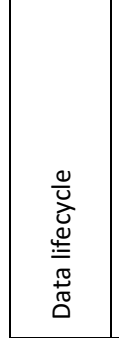 & 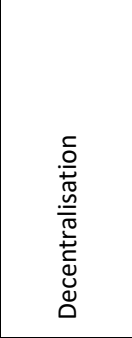 & 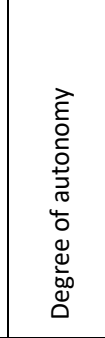 & 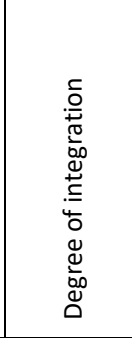 & 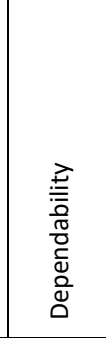 & 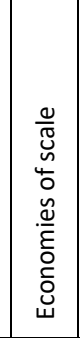 & 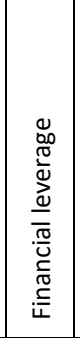 & 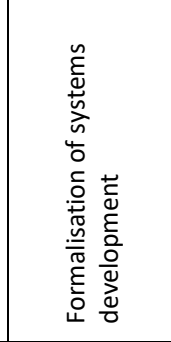 & 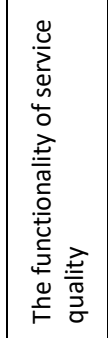 & 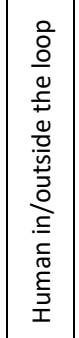 & 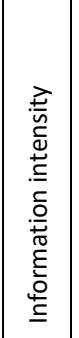 & 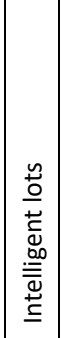 & 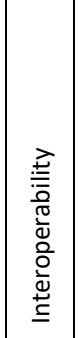 & 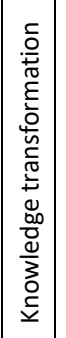 & 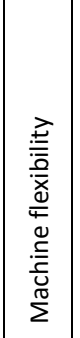 & 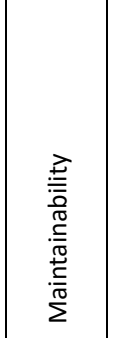 & 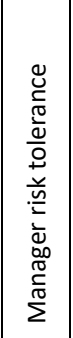 \\
\hline
\end{tabular}




\begin{tabular}{|c|c|c|c|c|c|c|c|c|c|c|c|c|c|c|}
\hline 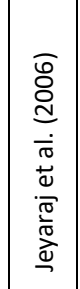 & 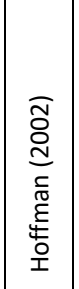 & 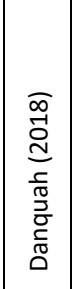 & 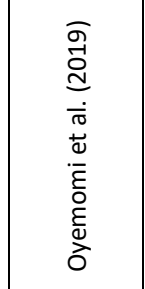 & 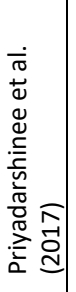 & 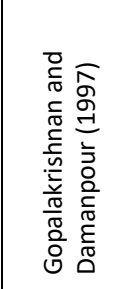 & 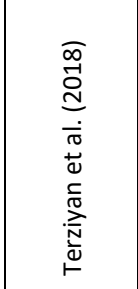 & 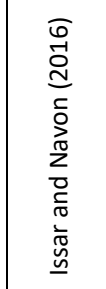 & 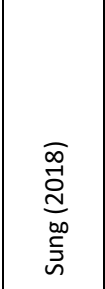 & 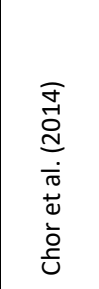 & 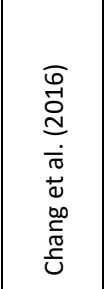 & 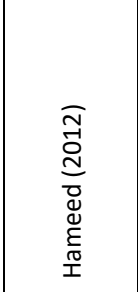 & 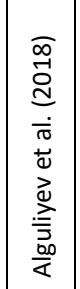 & 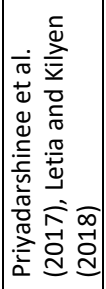 & 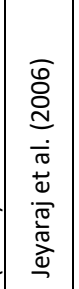 \\
\hline 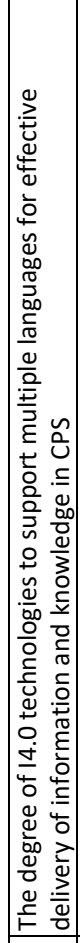 & 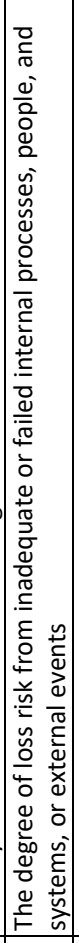 & 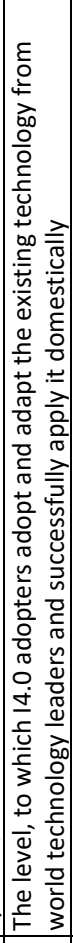 & 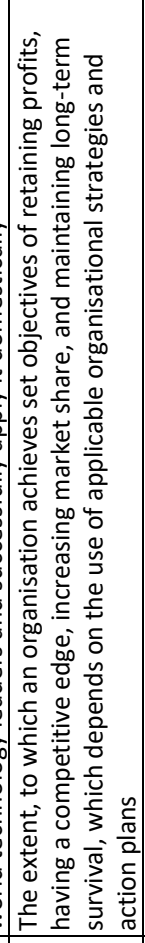 & 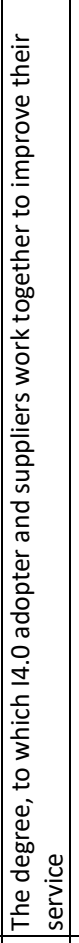 & 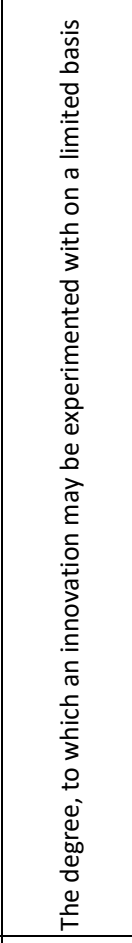 & 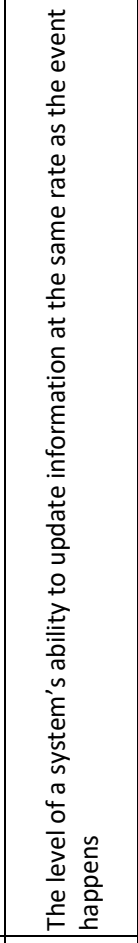 & 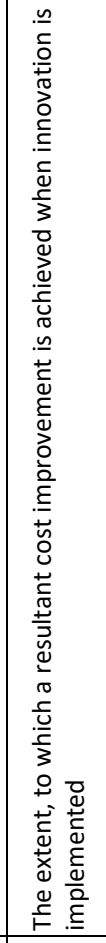 & 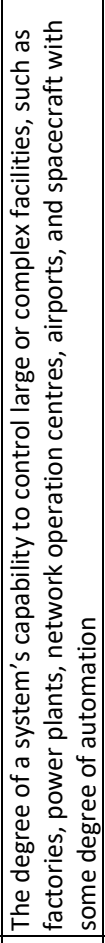 & 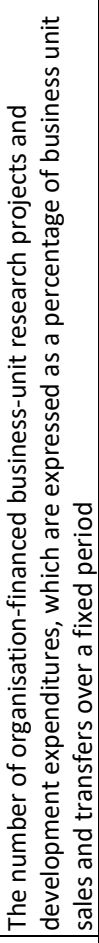 & 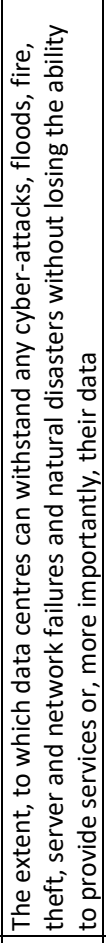 & 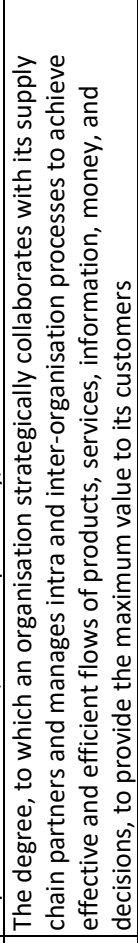 & 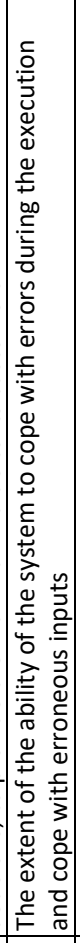 & 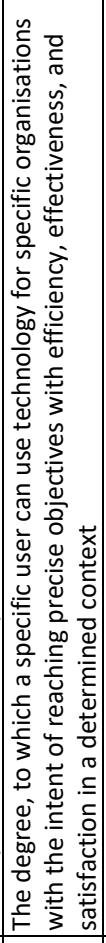 & 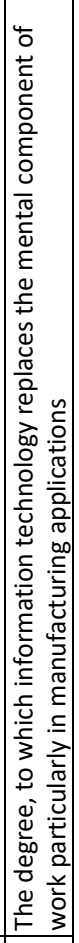 \\
\hline 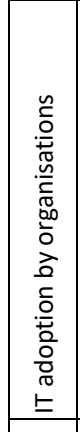 & & 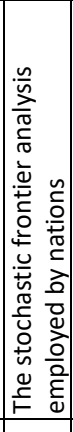 & 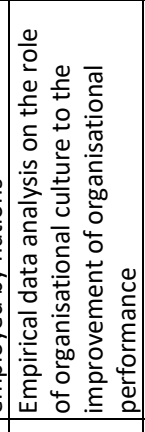 & 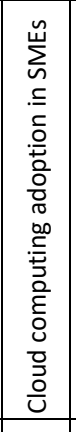 & 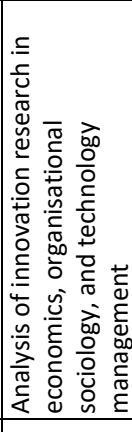 & 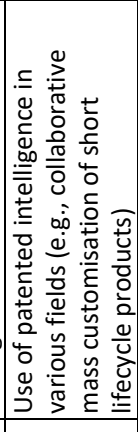 & 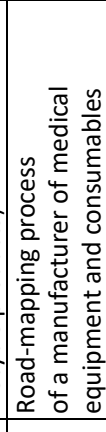 & 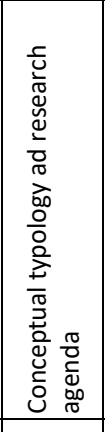 & $\begin{array}{l}\frac{0}{0} \\
0 \\
0 \\
0 \\
0 \\
0 \\
0 \\
0 \\
0 \\
0\end{array}$ & 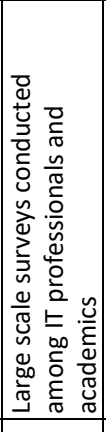 & 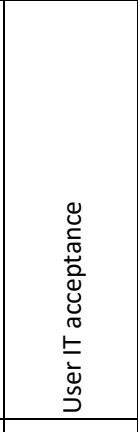 & 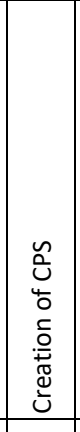 & 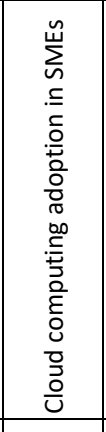 & 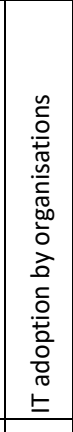 \\
\hline 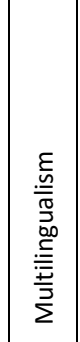 & 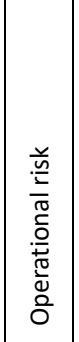 & 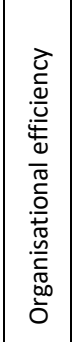 & 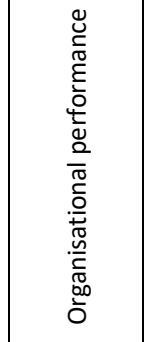 & 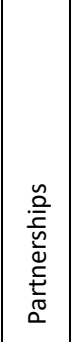 & 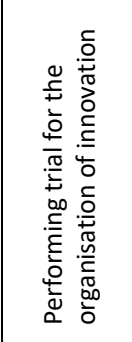 & 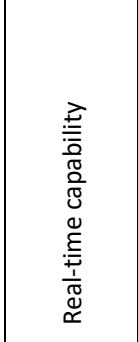 & 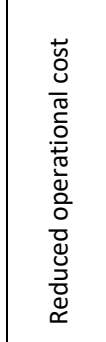 & 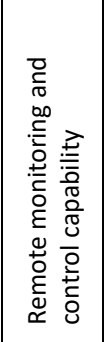 & 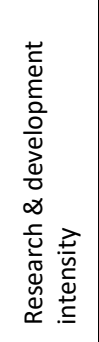 & 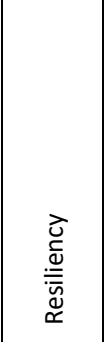 & 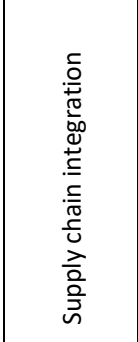 & 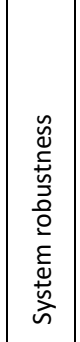 & $\bar{a}$ & \\
\hline
\end{tabular}




\section{INDICATORS FOR EACH STAGE OF ADOPTION}

Many efforts were put into establishing the indicators of innovation, which resulted in a long endeavour in the domain of application, reflected in the relevant literature (Moore \& Benbasat, 1991; Song et al., 2014). Notably, these indicators are used to answer the following questions: (i) how aware is a potential adopter of the innovation, and how is the innovation perceived? (ii) how ready is a potential adopter for the innovation and will the innovation be accepted?, and (iii) will an adopter continue the innovation if it is accepted? It can be inferred that the literature primarily revolves around these three questions. As such, the questions result lead to the stages of innovation adoption. In the literature, several scholars emphasise that innovation adoption is a process rather than an event, and different concerns may be predominant at different stages; hence, categorising the innovation adoption at such stages become relevant (Hameed et al., 2012). In this paper, the stages of adoption are categorised as pre-adoption, adoption, and post-adoption, together with respective identified indicators. Note that the indicators presented in this work are not directly measurable because they use metrics for the basic unit of measurement. Thus, an indicator may be represented by more than one metric depending on the firm or the industry under consideration. The context of the I4.0 implementation involves several cutting-edge technologies (e.g., sensor technology, robotics, etc.). However, these technologies are not explicitly represented by these indicators as they are considered as metrics in the proposed indicator system framework. Note that identifying the metrics for each indicator is out of the scope of this work. These metrics are highly casedependent and proposing such metrics may limit the flexibility of the proposed framework.

\subsection{Pre-Adoption STAge}

It can be seen that the first question stated previously underlies the pre-adoption stage. The preadoption stage involves all other conditions needed for the adoption of innovation before the evaluation of the decision is made to adopt or reject the innovation (Miranda et al., 2016). As Rogers (1995) puts it, the pre-adoption stage involves the previous conditions for adoption, knowledge or awareness of the innovation, and the perception of the potential adopter by acquiring more profound knowledge on the innovation. Inherently, adoption decisions usually come from information acquisition periods, which is implicit in technological innovations (Dimara \& Skuras, 2003). In the relevant literature, several scholars infer that the lack of awareness of innovation may explain the reason why its widespread adoption does not occur (Dimara \& Skuras, 2003).

The degree of awareness of I4.0 can be acquired depending on the attitude of stakeholders towards the innovation. One of the reasons why attitude plays a vital role in determining the degree of awareness is because it dictates the optimism or pessimism of potential adopters (Kerschner \& Ehlers, 2016). One of the most straightforward indicators that show the awareness of I4.0 is the perception of the term "Industry 4.0" (Priyadarshinee et al., 2017). Although the literature offers no strong support to the way the term used to describe an innovation affects its adoption, several papers consider the name significant in creating different perceptions or self-concepts (Garwood et al., 1980). On the other hand, perceived usefulness, perceived ease of use, relative advantage, trialability, observability, compatibility, and complexity, are indicators strongly supported in the literature and related to the perception of potential adopters (Rogers, 1995).

For instance, the perceived relative advantage is considered a sine qua non or necessary for adoption (Greenhalgh et al., 2004). If users do not perceive a relative advantage of innovation, it is generally not adopted (Rogers, 1995). However, although considered to be critically important, it does not guarantee widespread adoption, thus, suggesting the need to look into other factors (Greenhalgh et al., 2004). Trialability - the extent, at which an innovation can be tried on a limited basis - is also strongly supported by many scholars in the literature (Miranda et al., 2016). It is strongly argued that although being able to test the innovation on a smaller sample space does not guarantee success when applied at a larger scale, it increases the confidence of adopters in the innovation (Plsek, 2003). Rogers (1995) argues that it is positively linked to the adoption of an innovation.

Aside from directly testing the innovation, observing the innovation already adopted by others may also affect the perception of potential adopters (Miranda et al., 2016). Potential adopters use a riskreduction strategy of seeking information from others who have already adopted the innovation of interest because adopters are usually faced with uncertainty about the consequences of their deci- 
sions, which contributes to their perceived risk (Mehrad \& Mohammadi, 2017). It must be noted that trialability and observability are different. Trialability involves direct testing of innovation, and observability involves indirect testing of the innovation through others who have adopted the innovation.

Similarly, another important indicator of innovation adoption is compatibility or the degree of how accustomed an innovation is to existing standards, norms, and values of potential adopters (Greenhalgh et al., 2004). Many scholars argue that compatibility may significantly predict whether innovation will be accepted or not (Greenhalgh et al., 2004). For instance, if a government agency intends to make their citizens use services online, they must provide information and services in a manner that is consistent with other ways citizens have dealt with the government, e.g., online forms should resemble paper forms that citizens are familiar with (Rogers, 1995; Joia et al., 2016). If innovation results in actions that are very different from the existing practice, potential adopters perceive it as risky, thus, possibly rejecting the innovation (Joia et al., 2016). Likewise, if innovation is perceived to be highly sophisticated, potential adopters will likely reject it (Agarwal \& Prasad, 1998; Greenhalgh et al., 2004). A complex system is one that cannot be broken down into manageable parts. Several scholars claim that most organisations that opt for innovation operate in such a manner if not appropriately managed; thus, they may cause a negative perception for potential adopters (Szczerbicki, 2008). To this end, complexity plays a crucial role in indicating the perception of potential I4.0 adopters.

Several scholars also point out the importance of communication channels used in spreading information about the innovation, which contributes to the perception of potential adopters (Agarwal \& Prasad, 1998). This result was found to be significant by scholars in relevant fields (Adegbola \& Gardebroek, 2007). Mainly, Adegbola \& Gardebroek (2007) found that when the information about the innovation was spread through external sources (e.g., knowledgeable external sources) adopters tended to have a more favourable perception of the innovation than when it was spread through internal sources (e.g., adopters who were still in the process of adoption). Hence, the perceptions of potential I4.0 adopters can be affected by the flow experience of I4.0, the emergence of global distribution networks, and some information sources.

The pre-adoption stage is concerned mainly with perceptions of potential adopters, which result from acquired awareness and more profound knowledge of I4.0. Technological innovativeness, unlike the indicators mentioned above, is more of an inherent characteristic of potential adopters rather than one generated as a result of acquired awareness. Moreover, it plays a vital role in connecting the pre-adoption (perception) indicators to the adoption (persuasion) indicators of I4.0 (Agarwal \& Prasad, 1998).

\subsection{Adoption STAgE}

The adoption stage encompasses the period, in which the decision unit is engaged in activities that lead to the choice to adopt or reject innovation, otherwise known as the decision stage (Miranda et al., 2016). In contrast to the pre-adoption stage, the adoption stage involves the persuasion phase of the organisation to decide if innovation must be adopted or rejected (Miranda et al., 2016). In other words, it comprises activities (e.g., financial, technical, and strategic) that evaluate the readiness of systems to implement I4.0 in an organisation (Hameed et al., 2012). This section discusses the adoption stage indicators.

Financial evaluation activities are a straightforward indicator of the readiness for innovation (Quevedo et al., 2017). It is common practice for managers to evaluate the risks of innovation projects, mainly financial risks, since they usually may cause the failure of some innovation projects (Pellegrino \& Savona, 2017). Also, risks, costs, and uncertainties are weight against benefits and incentives that would be gained by the organisation that implements innovation (Chor et al., 2014). Such activities are practical ways used by organisations to analyse the desirability of innovation (Prest \& Turvey, 1965).

Several scholars point out the importance of leadership and support in the decision to adopt innovations (Greenhalgh et al., 2004). In particular, this mostly relates to CEO advocacy (Chor et al., 2014). The alignment between innovation and prior organisational goals makes the adoption more likely (Greenhalgh et al., 2004). Some scholars maintain that innovation adoption is more probable when key individuals (e.g., CEO) are willing to support innovation in their social networks (Greenhalgh et al., 2004). Likewise, the organisation must also have the technical capability to evaluate innovation (Boh et al., 2014). To this end, the presence of technical support and expertise, as well as IS infrastructures, are important indicators of an organisation evaluating its readiness for I4.0 (Hameed et al., 2014). 
As already mentioned, an organisation must be capable of the successful adoption of innovation. However, not only it needs technical capability but also the expertise to deal with its market environment (Zhang \& Hartley, 2018). As such, both the level of competitive pressure and the level of customer interaction are useful indicators for the adoption decision (Priyadarshinee et al., 2017). Several scholars maintain that the level of competitive pressure is an implicit consequence of the accelerated competitive environment, primarily due to the desire to create new products and processes in an improvised manner (Zhang \& Hartley, 2018). Subsequently, scholars claim that the level of customer interaction is a critical determinant of the organisational performance; thus, also important for the adoption decision (Manral, 2010).

The geographic location of an organisation also plays a vital role in indicating the status of the adoption decision. Several scholars claim that geographical proximity is a necessary condition in the diffusion of knowledge or innovation (Martinez-Noya \& Garcia-Canal, 2017). It is, thus, widely accepted that determining the geographic location of an organisation is a first-order strategic decision of stakeholders (Escuer et al., 2014). On the other hand, the reputation of suppliers is an important indicator at the decision stage. Since I4.0 promises new products and process innovations, potential adopters ensure that during the implementation stage, they would not face the risk of knowledge leakage. Knowledge leakage becomes a potential problem in at least two ways, i.e. (i) when suppliers serve a competitor who puts the organisation at the risk of knowledge spill-over in favour of the competitor, and (ii) when the supplier becomes a potential competitor due to the knowledge spill-over (Martinez-Noya \& Garcia-Canal, 2017). Moreover, scholars argue that such risk is higher at locations with weak intellectual property (IP) protection.

\subsection{Post-Adoption Stage}

The post-adoption stage occurs when a decision unit puts the technology in use (Rogers, 1995). That is, a decision unit, as in an organisation, finally implements the technology and correspondingly evaluates the advantages and disadvantages of technology adoption, which in turn, guides organisations in their decision of whether such adoption should be continued or not. Such action boosts the efficiency of an organisation, given its successful application of new technology in the local context as well as its capability to compete according to strategies and action plans (Oyemomi et al., 2019). This adoption decision, however, is subject to a certain degree of integration depending on the available resources and risk aversion of an organisation (Hameed, 2012). When an implemented technology is perceived to be riskier, an organisation's willingness to continue the adoption may correspondingly diminish.

In the case of the I4.0 adoption, it is a basic necessity to ensure open access to critical technologies, such as IoT, CPS, smart manufacturing, and cloud computing (Priyadarshinee et al., 2017). When organisations have a sense of ownership stake in one or more critical I4.0 technologies, the eventual postadoption of such technology becomes more attractive. Otherwise, the acquisition of these technologies may potentially delay the I4.0 implementation on the grounds of economic risk barriers, financial leverage, and functionality of service quality offered by the new technology amidst the competition in the supply chain network (Joachim et al., 2018).

Also, the amount of operational cost reduced in the implementation of I4.0 must be deemed reasonable for organisations to continue using the technology (Lee et al., 2015). When representations of cost reduction as in pay-per-usage and reduced facilities are substantial enough to warrant a continued adoption of technology, organisations can be further driven to do so. The same inference can also be drawn for economies of scale (Priyadarshinee et al., 2017) and work simplification (Jeyaraj, 2006).

An organisation that implemented I4.0 is expected to have a system that is adaptive by plugand-work mechanism (Monostori et al., 2016), autonomous (Letia \& Kilyen, 2018), decentralised (Terziyan et al., 2018), dependable (Alguliyev et al., 2018), interoperable (Priyadarshinee et al., 2017), capable of real-time operations (Terziyan et al., 2018), remote monitoring and control (Sung, 2018), resilient (Chang et al., 2016), robust (Alguliyev et al., 2018), capable of handling full information (Sabherwal \& King, 1991), flexible (Browne et al., 1984), capable of maintaining data lifecycle (Tao et al., 2018), usable (Priyadarshinee et al., 2017), and with intelligent lots (Li et al., 2012). Given that such capabilities are made possible by critical I4.0 technologies, an organisation is more likely to confirm the continued adoption of such technology, based on trials when its performance meets prior expectations. That is, a positive assessment should be observed with the benefits outweighing the issues arising from the adoption of I4.0 
(Miranda et al., 2016). Such issues may include maintainability (Blanchard et al., 1995), multilingualism (Jeyaraj et al., 2006), and exposure to operational risk (Hoffman, 2002). In such a case, the new technology proceeds to be institutionalised and part of the daily operations of the adopting organisation (Rogers, 1995). Otherwise, a probably discontinued adoption, if not withdrawal, of the technology may be decided when an organisation perceives more inhibited changes in the transformation process (Lienert, 2015).

Other strategic activities, such as the formalisation of systems development (Mathiassen \& MunkMadsen, 2007), knowledge transformation (Chor et al., 2014), a culture of change (Lienert, 2015), customer co-creation (Sung, 2018), intense research and development (Chor et al., 2014), and partnership establishment (Priyadarshinee et al., 2017), are also considered as indicators of this post-adoption phase. While the I4.0 adoption continues, it is imperative for organisations to regularly align their goals to a prescribed implementation on the following: (1) required brand and methodology specifications, (2) ideal product and project developments, (3) perceived change in the demands of core tasks, (4), open innovation among organisations and customers, (5) substantial efforts of generating innovative ideas, and (6) strong ties within a network of suppliers. As a result, straightforward transparency among customers and just the involvement of humans (i.e., operators or workers) in the loop may be upheld.

In summary, sets of indicators for each stage are positioned according to relevance as stipulated by a robust guideline. This guideline is streamlined like a conventional decision-making process where actions are preceded by the process of assessment and selection. In the context of the I4.0 implementation, this process is broken down into three segments, that is, perception, decision, and implementation, represented by the three stages of adoption. The preadoption stage encompasses indicators relevant to the perception of stakeholders as to the level or status of an organisation's I4.0 technology adoption. This apprehension is influenced by the knowledge and understanding of the involved personage about technologies that fall under the concept of Industry 4.0. This stage also includes the evaluation of the advantages and disadvantages of potentially implementing or rejecting I4.0 technology. The adoption stage includes indicators that discuss the decision-making process undergone by stakeholders in their conjecture for the potential to adopt or reject the technolo- gies highlighted at the previous (pre-adoption) stage. The suitability shapes the final decision regarding the technology in terms of the fit for the organisation's needs and goals. Going from one stage to the next, the scope of the decision-making process becomes more complex. Moreover, at this stage, indicator sets provide a detailed outlook on being able to initially distinguish the capabilities of an innovation that tailor to the organisational needs. The post-adoption stage mainly concerns indicators that aided in confirming the initial evaluation set at previous stages and, thus, provide insights on the likelihood to continue the implemented innovation, or otherwise, end its use when proven to be depreciatory.

\subsection{FINAL NOTE ON INDICATOR SETS FOR STAGES OF ADOPTION}

In summary, there are 11 indicators at the preadoption stage, 14 indicators at the adoption stage, and 37 indicators at the post-adoption stage. Every stage of adoption has a different number of indicators, which signifies its outright position in terms of stage suitability. In terms of a stage, suitability means that indicator sets are assigned to the most appropriate stage where a thorough management dashboard is deemed most necessary. It is also important to emphasise that this paper aims to present a set of indicators for every stage of adoption concerning its function and contextual representation rather than evaluate indicators at each stage quantitively. Some indicators need to be firmly established at a particular stage, so that its implicit representation in succeeding stages may already be covered. As an illustration, take, for example, the following indicators of the preadoption stage: the perception of the term Industry 4.0 , perceived ease of use, and observability. It is canonical for business stakeholders to be able to initially distinguish the capabilities of innovation before making the adoption decision (i.e., adoption stage) and its eventual implementation (i.e., post-adoption stage). Following this principle, indicators - such as the perception of the term Industry 4.0, perceived ease of use, and observability - are believed to be most suitable for the pre-adoption stage since they represent the knowledge or awareness of innovation as well as the perception of stakeholders. This compels stakeholders to create an effective management dashboard based on such indicators and other strategic data that comes with it at this stage. Furthermore, it is necessary to understand that as stages of adoption progress, the scope of decision-making attributed to 
stakeholders becomes vast and even more complex due to the implied transfer and continuous management of tasks embedded in each indicator. That is, despite a unique set of indicators in succeeding (i.e., adoption and post-adoption) stages, it is nevertheless suggestive of the continued attention to the indicators of the previous stage.

\section{PROPOSED APPLICATIONS}

The applicability of the proposed indicator sets is demonstrated by two case studies of two leading manufacturing firms in the Philippines. Generally, indicators are developed to manage and plan a company's operational performance appropriately. Conversely, there have been several aggregation methods developed to come up with a single score aggregate index present in the extant literature, such as the fuzzy Delphi method (FDM), analytic hierarchy process (AHP), analytic network process (ANP), simple additive weighting method (SAW), decision making trial and evaluation laboratory (DEMATEL), the technique for the order of prioritisation by similarity to ideal solution (TOPSIS), aggregated indices randomisation method (AIRM), etc. In the context of this study, the LWAA operator method was used to arrive at an overall performance index of a firm's status on the I4.0 adoption. Aiming to maintain the generality in the list of indicators, all indicators were assumed to have equal weights, although this assumption may not hold in practice. Assigning weights of all indicators is out of the scope of this work. To assign weights to indicators, firms may adopt a prioritisation process.

The following sections present the two case studies. Each case focuses on firms that have adopted a new form of technology in their production processes.

\subsection{CASe STUdy 1}

The first case study was performed at a premier designer and manufacturer of electronic components for mobile communications and consumer electronics, producing microphones, speakers, and medical hearing devices. The company has been introducing disruptive technologies for over 65 years and is one of the industry leaders at present. Its strong drive for continuous improvement has pushed the firm to acquire and integrate innovative concepts and new forms of technology in its manufacturing processes. For the past two years, it has adopted the concept of IoT in its products to further enhance user experience. Also, it has ongoing plans for the implementation of smart manufacturing in incoming brands. Consequently, it can be argued that the firm has had a proper understanding of I4.0 and its implementation. To demonstrate the application of the proposed innovation stages and actual industry implications, this section presents the results of the evaluation of company A.

For company A, a sample research questionnaire used at the pre-adoption stage is presented in Table 3, further showing the linguistic ratings given by respondents for each indicator on the list. Subse-

Tab. 3. Sample pre-adoption stage questionnaire for company A

\begin{tabular}{|c|c|c|c|c|c|c|}
\hline \multicolumn{7}{|c|}{ COMPANY A } \\
\hline No. & INDICATOR & $\begin{array}{c}\text { VERY } \\
\text { POOR } \\
\text { (VP) }\end{array}$ & $\begin{array}{l}\text { POOR } \\
\text { (P) }\end{array}$ & $\begin{array}{c}\text { FAIR } \\
\text { (F) }\end{array}$ & $\begin{array}{c}\text { GOOD } \\
\text { (G) }\end{array}$ & $\begin{array}{c}\text { VERY } \\
\text { GOOD } \\
\text { (VG) }\end{array}$ \\
\hline 1. & Compatibility & & & & & $\checkmark$ \\
\hline 2. & Perceived ease of use & & & & $\checkmark$ & \\
\hline 3. & Perceived usefulness & & & & & $\checkmark$ \\
\hline 4. & Flow experience & & & & $\checkmark$ & \\
\hline 5. & Internal information sources & & & & $\checkmark$ & \\
\hline 6. & External information sources & & & & $\checkmark$ & \\
\hline 7. & Observability & & & & & $\checkmark$ \\
\hline 8. & Perceived risk & & & $\checkmark$ & & \\
\hline 9. & Perception of the term Industry 4.0 & & & & & $\checkmark$ \\
\hline 10. & Subjective importance of tasks & & & & $\checkmark$ & \\
\hline 11. & Technological innovativeness & & & & & $\checkmark$ \\
\hline
\end{tabular}


Tab. 4. Sample post-adoption stage questionnaire for company B

\begin{tabular}{|c|c|c|c|c|c|c|}
\hline \multicolumn{7}{|c|}{ COMPANY B } \\
\hline No. & INDICATOR & $\begin{array}{c}\text { VERY } \\
\text { POOR } \\
\text { (VP) }\end{array}$ & $\begin{array}{l}\text { POOR } \\
\text { (P) }\end{array}$ & $\begin{array}{c}\text { FAIR } \\
\text { (F) }\end{array}$ & $\begin{array}{c}\text { GoOD } \\
\text { (G) }\end{array}$ & $\begin{array}{c}\text { VERY } \\
\text { GOOD } \\
\text { (VG) }\end{array}$ \\
\hline 1. & Access to 14.0 technologies & & & & & $\checkmark$ \\
\hline 2. & Adaptivity by plug-and-work & & & & $\checkmark$ & \\
\hline 3. & Auditability & & & & $\checkmark$ & \\
\hline 4. & Culture of change & & & & & $\checkmark$ \\
\hline 5. & Customer co-creation & & & & $\checkmark$ & \\
\hline 6. & Data lifecycle & & & & $\checkmark$ & \\
\hline 7. & Decentralisation & & & & & $\checkmark$ \\
\hline 8. & Degree of autonomy & & & & $\checkmark$ & \\
\hline 9. & Degree of integration & & & & $\checkmark$ & \\
\hline 10. & Dependability & & & & & $\checkmark$ \\
\hline 11. & Economies of scale & & & & & $\checkmark$ \\
\hline 12. & Financial leverage & & & & & $\checkmark$ \\
\hline 13. & Formalisation of systems development & & & & & $\checkmark$ \\
\hline 14. & The functionality of service quality & & & & & $\checkmark$ \\
\hline 15. & Human in/outside the loop & & & & & $\checkmark$ \\
\hline 16. & Information intensity & & & & & $\checkmark$ \\
\hline 17. & Intelligent lots & & & & $\checkmark$ & \\
\hline 18. & Interoperability & & & & & $\checkmark$ \\
\hline 19. & Knowledge transformation & & & $\checkmark$ & & \\
\hline 20. & Machine flexibility & & & $\checkmark$ & & \\
\hline 21. & Maintainability & & & & & $\checkmark$ \\
\hline 22. & Manager risk tolerance & & & & & $\checkmark$ \\
\hline 23. & Multilingualism & & & & & $\checkmark$ \\
\hline 24. & Operational risk & & & & & $\checkmark$ \\
\hline 25. & Organisational efficiency & & & & & $\checkmark$ \\
\hline 26. & Organisational performance & & & & & $\checkmark$ \\
\hline 27. & Partnerships & & & & & $\checkmark$ \\
\hline 28. & Performing trial for the organisation of innovation & & & & & $\checkmark$ \\
\hline 29. & Real-time capability & & & & $\checkmark$ & \\
\hline 30. & Reduced operational cost & & & & $\checkmark$ & \\
\hline 31. & Remote monitoring and control capability & & & & $\checkmark$ & \\
\hline 32. & Research \& development intensity & & $\checkmark$ & & & \\
\hline 33. & Resiliency & & & & & $\checkmark$ \\
\hline 34. & Supply chain integration & & & & & $\checkmark$ \\
\hline 35. & System robustness & & & & & $\checkmark$ \\
\hline 36. & Usability & & & & & $\checkmark$ \\
\hline 37. & Work simplification & & & & & $\checkmark$ \\
\hline
\end{tabular}


quently, based on Xu (2006), the respondent answers were coded into five extended discrete linguistic labels expressed in equation (2). Then, to come up with a single score aggregate index for each stage, equation (3) was used. For the pre-adoption stage, using the proposed procedure presented in Section 3.3, company A attained an overall score of 1.364 , signifying a good performance. Meanwhile, the adoption and post-adoption stages achieved good and fair scores of 1.643 and 0.784 , respectively. These ratings imply that initiatives performed by the firm are aligned with the performance of each indicator concerning the implementation of I4.0 technologies. Since company A has a fair adoption performance, it needs to devise a holistic strategy to enhance its performance. Table 3 also provides insights into the performance of company A in terms of each indicator. Of the 11 indicators, the indicator of perceived risk has the lowest performance value, which implies that company A has limited risk assessment efforts in the adoption of I4.0 technologies. Thus, a thorough assessment of risks in various sources must be implemented to improve its pre-adoption status.

Furthermore, the evaluation provides insights into areas of improvement for the company A. By dichotomising each indicator, company A may adopt insights into its decision-making process, resource allocation decisions, and strategy formulation. However, these results must be considered with caution as some limitations in the evaluation process exist. First, just for the sake of demonstrating the applicability of the proposed indicator system, the case study only focuses on a small group of decision-makers who performed the linguistic evaluation process.For a more rigid application, an evaluation must be carried out holistically and involve a well-represented group of decision-makers from among all company stakeholders. Second, the evaluation is rough, as more quantifiable metrics were not determined for each indicator. Metrics could provide more meaningful and realistic measurements of indicators, which would provide a clear understanding of performance in terms of the I4.0 adoption. Third, coming up with a single-valued index, weights of indicators are considered equal. It is straightforward to note that each indicator has a varying degree of importance to a stage of the I4.0 adoption. Thus, having indicators of equal weights is just an oversimplification of complex real-life decision-making. Stakeholders may collectively assign a weight for each indicator based on its importance for an I4.0 adoption stage.

\subsection{CASE STUdY 2}

The second case study focused on a leading supplier of automotive seating solutions and electrical distribution systems and architectures. Products of this company have consistently delivered an elevated automotive experience for the end-users. As a global business that has been in the market for more than a century, it has continually achieved excellence by rigorously adapting to new technologies. To date, it has adopted the concept of IoT and smart manufacturing in its production processes to achieve a smoother flow of materials and workforce from the dock to dock. The extensive experience of the company in acquiring and implementing innovative strategies makes it another suitable source for the verification of the practical relevance of indicator sets.

Ratings issued by company B are presented in Table 4. Using the same procedure as with company A, the respondent's answers were coded as in equation (2). For the computation of the overall performance, equation (3) was used. As for the post-adoption stage, the overall index had a value of 1.541 , which denoted a good implementation performance. Thus, indicators of this stage of the I4.0 implementation are well-evident at the firm. However, similar implications and precautions of the results, as discussed in the case study 1, are also applicable in this case. For brevity, these discussions are not presented.

\section{CONCLUSIONS}

While innovation strategies in globalisation domains have been modelled after different stages of adoption, unfortunately, the implementation of Industry 4.0 has not been established in the same way despite being under the agenda of innovation. Due to the multi-phase and multi-dimensional nature of innovations in general, it is imperative to put more emphasis on various stages of adoption, so that dominant issues arising distinctly from each stage could be addressed more responsively by firms using programmes and initiatives. Furthermore, providing such a holistic approach embedded in the innovation process can potentially prevent haphazard implementation, poor resource allocation, and a myopic view of I4.0. Among other approaches in the holistic evaluation of the innovation process, the development of indicator sets is deemed by stakeholders a crucial step towards effective decision-making, 
resource allocation decisions, and strategy and policy formulation. Quantifiable sets of indicators allow managers and decision-makers to keep track of their I4.0 performance and strategically plan and direct initiatives to progress more efficiently and effectively.

Thus, this work intends to close two critical gaps in the literature: (a) to treat I4.0 as the innovation process that has specific adoption stages, and (b) to develop an indicator set for each stage. After a comprehensive review of related literature, 62 indicators are extracted: 11 for the pre-adoption stage, 14 for the adoption stage, and the remaining 37 for the postadoption stage. The distribution of indicators according to the adoption stage was based on its operational description and its relations to the innovation process. Indicators at each I4.0 stage were intended to describe the status of an organisation in its path towards the I4.0 adoption as well as serve as dashboards in gauging the organisation's direction and its speed of implementation. Note that the status that can be generated from the indicators is the performance of an organisation in discrete time rather than continuous. Thus, these proposed indicators provide a picture of an organisation at a particular stage and specific time.

The pre-adoption indicators are mostly generated by the perception of potential adopters except for one indicator (i.e., technological innovativeness), which depends primarily on the attitude while adoption indicators relate to the evaluation activities of potential adopters concerning their financial, technical, and organisational capabilities. Note that the majority of the identified indicators belong to the post-adoption stage. This finding implies that this stage critically shapes the decision-makers in finally implementing the technology and evaluating the advantages and disadvantages of adoption. Furthermore, indicators in this stage dictate whether the adoption is continued or not, depending on how the desirability of benefits outweighs the severity of risks, or vice-versa.

With the proposed I4.0 indicators, some possible applications can be described. First, indicators may help organisations establish strategic plans, both short-term and long-term. At the pre-adoption and adoption stages, stakeholders may implement organisation-wide information campaigns, so that all levels of the organisation may better understand the role of technologies integrated into I4.0 as well as its capability to bank on the empowerment of organisations in identifying opportunities for the I4.0 implementation. At the post-adoption stage, resources can be allocated appropriately so that critical indicators can be sufficiently supported. Second, indicators promote a platform for performance evaluation. With the use of these indicators at various stages, managers and decision-makers can reflect the organisation's performance and eventually produce inputs in planning initiatives and strategies. Additionally, performance evaluation can be served as inputs in employee reward systems. Third, establishing indicators may leave open areas of improvement that must be addressed to adopt I4.0 successfully. On the supply side, such indicators may serve as metrics for hotspots to inform the developers of I4.0 (e.g., CPS, IoT, etc.) about the ease-of-adoption of I4.0. For instance, if most potential adopters have a high score for one indicator, developers may modify the technologies used in I4.0, so that they become manageable, hence, increasing the chance of adoption. Finally, the proposed I4.0 indicators can serve as inputs to balanced scorecards and performance dashboards. This application is particularly relevant to the post-adoption stage, where managers can monitor the performance of their organisation in light of these indicators.

While two case studies were presented in this work to shed more light on the use of I4.0 indicators, such demonstration has some limitations. First, performance ratings are desirable from more involved focus-group discussions at various organisational levels. In the case studies, middle-managers were only asked to provide ratings that may not reflect the perspectives of upper management and first-line management. Thus, involving the perspectives of different organisational levels may provide a more accurate reflection of organisational performance. Second, although the indicators project quantifiable measurement concepts, there is room to entertain the idea that defining each indicator with finer metrics would provide higher resolutions of organisational performance in terms of I4.0. Thus, managers and decision-makers could establish metrics for each indicator relevant to a specific industry. Third, in the case studies, the assumption that I4.0 indicators have equal priority weights does not reflect real-life conditions. It is plausible to note that I4.0 indicators play varying roles to a certain degree so that the establishment of priority weights for each indicator is much desired. Managers, with the aid of analysts, may adopt any suitable prioritisation methods such as simple additive weighting (SAW), analytic hierarchy process (AHP), best-worst method (BWM), etc. With the implementation of these methods, the role of each indicator in I4.0 adoption may be better highlighted. 
Finally, an indicator system that contains a rigorous framework for a composite index can be implemented by managers and decision-makers. Indicator systems can be structured in a hierarchy to generate a composite index that describes the overall performance of an organisation at a particular adoption stage. This information regarding the overall performance provides a macro view on the status of the I4.0 adoption stage, which can be used by organisations for monitoring and comparison with other market players.

\section{LITERATURE}

Adegbola, P., \& Gardebroek, C. (2007). The effect of information sources on technology adoption and modification decisions. Agricultural Economics, 37(1), 5565. doi: 0.1111/j.1574-0862.2007.00222.x

Aduda, K., Thomassen, T., Zeiler, W., Labeodan, T., Boxem, G., van der Velden, J., \& Dubbeldam, J. W. (2014). The human in the loop: An approach to individualize smart process control. Procedia Environmental Sciences, 22, 302-312. doi: 10.1016/j.proenv.2014.11.029

Agarwal, R., \& Prasad, J. (1998). The antecedents and consequents of user perceptions in information technology adoption. Decision Support Systems, 22(1), 1529. doi: 10.1016/S0167-9236(97)00006-7

Alekseev, A. N., Buraeva, E. V., Kletskova, E. V., \& Rykhtikova, N. A. (2018). Stages of Formation of Industry 4.0 and the Key Indicators of Its Development. Industry 4.0: Industrial Revolution of the 21st Century, 169, 93100. doi: 10.1007/978-3-319-94310-7_9

Alguliyev, R., Imamverdiyev, Y., \& Sukhostat, L. (2018). Cyber-physical systems and their security issues. Computers in Industry, 100, 212-223. doi: doi. org/10.1016/j.compind.2018.04.017

Almada-Lobo, F. (2016). The Industry 4.0 revolution and the future of manufacturing execution systems (MES). Journal of Innovation Management, 3(4), 1621. doi: 10.24840/2183-0606_003.004_0003

Arnold, C., Veile, J., \& Voigt, K. I. (2018). What drives industry 4.0 adoption? An examination of technological, organizational, and environmental determinants. 27th International Conference on Management of Technology (IAMOT), Birmingham, United Kingdom.

Attaran, M. (2017). The rise of 3-D printing: The advantages of additive manufacturing over traditional manufacturing. Business Horizons, 60(5), 677-688. doi: 10.1016/j.bushor.2017.05.011

Birchall, D., Chanaron, J. J., Tovstiga, G., \& Hillenbrand, C. (2011). Innovation performance measurement: Current practices, issues and management challenges. International Journal of Technology Management, 56(1), 1-20. doi: 10.1504/ijtm.2011.042492

Blanchard, B. S., Verma, D., \& Peterson, E. L. (1995). Maintainability: A key to effective serviceability and maintenance management. New York, United States: Wiley.
Bleicher, J., \& Stanley, H. (2016). Digitization as a catalyst for business model innovation a three-step approach to facilitating economic success. Journal of Business Management, 4(2), 62-71.

Boh, W. F., Evaristo, R., \& Ouderkirk, A. (2014). Balancing breadth and depth of expertise for innovation: A 3M story. Research Policy, 43(2), 349-366. doi: 10.1016/j. respol.2013.10.009

Bohnsack, R., \& Pinkse, J. (2017). Value propositions for disruptive technologies: Reconfiguration tactics in the case of electric vehicles. California Management Review, 59(4), 79-96. doi: 10.1177/0008125617717711

Borrás, S., \& Edquist, C. (2013). The choice of innovation policy instruments. Technological Forecasting and Social Change, 80(8), 1513-1522. doi: 10.1016/j.techfore.2013.03.002

Brettel, M., Friederichsen, N., Keller, M., \& Rosenberg, M. (2014). How virtualization, decentralization and network building change the manufacturing landscape: An Industry 4.0 Perspective. International Journal of Information and Communication Engineering, 8(1), 37-44.

Browne, J., Dubois, D., Rathmill, K., Sethi, S. P., \& Stecke, K. E. (1984). Classification of flexible manufacturing systems. The FMS Magazine, 2(2), 114-117.

Caiazza, R., \& Volpe, T. (2017). Innovation and its diffusion: Process, actors and actions. Technology Analysis \& Strategic Management, 29(2), 181-189. doi: 10.1080/09537325.2016.1211262

Castelo-Branco, I., Cruz-Jesus, F., \& Oliveira, T. (2019). Assessing Industry 4.0 readiness in manufacturing: Evidence for the European Union. Computers in Industry, 107, 22-32. doi: 10.1016/j.compind.2019.01.007

Cavdar, S. C., \& Aydin, A. D. (2015). An empirical analysis about technological development and innovation indicators. Procedia-Social and Behavioral Sciences, 195, 1486-1495. doi: 10.1016/j.sbspro.2015.06.449

Chang, V., Ramachandran, M., Yao, Y., Kuo, Y. H., \& Li, C. S. (2016). A resiliency framework for an enterprise cloud. International Journal of Information Management: The Journal for Information Professionals, 36(1), 155-166. doi: 10.1016/j.ijinfomgt. 2015.09.008

Chor, K. H. B., Wisdom, J. P., Olin, S. C. S., Hoagwood, K. E., \& Horwitz, S. M. (2014). Measures for predictors of innovation adoption. Administration and Policy in Mental Health and Mental Health, 42(5), 545-573. doi: 10.1007/s10488-014-0551-7

Christensen, C. M., Bartman, T., \& van Bever, D. (2016). The hard truth about business model innovation. Retrieved from http://sloanreview.mit.edu/article/thehardtruth-about-business-model- innovation/

Crossan, M. M., \& Apaydin, M. (2010). A multi-dimensional framework of organizational innovation: A systematic review of the literature. Journal of Management Studies, 47(6), 1154-1191. doi: 10.1111/j.1467-6486.2009.00880.x

Damanpour, F., \& Schneider, M. (2006). Phases of the adoption of innovation in organizations: Effects of environment, organization and top managers 1. British Journal of Management, 17(3), 215-236. doi: 10.1111/j.1467-8551.2006.00498.x 
Danquah, M. (2018). Technology transfer, adoption of technology and the efficiency of nations: Empirical evidence from sub Saharan Africa. Technological Forecasting and Social Change, 131, 175-182. doi: 10.1016/j.techfore.2017.12.007

Datta, A., Mukherjee, D., \& Jessup, L. (2015). Understanding commercialization of technological innovation: Taking stock and moving forward. R\&D Management, 45(3), 215-249. doi: 10.1111/radm.12068

Datta, A., Reed, R., \& Jessup, L. (2013). Commercialization of innovations: An overarching framework and research agenda. American Journal of Business, 28(2), 147-191. doi: 10.1108/AJB-08-2012-0048

Davis, F. D. (1989). Perceived usefulness, perceived ease of use, and acceptance of information technology. MIS Quarterly, 13(3), 319-340. doi: 10.2307/249008

De Sousa Jabbour, A. B., Jabbour, C. J., Foropon, C., \& Godinho Filho, M. (2018). When titans meet - Can industry 4.0 revolutionise the environmentally-sustainable manufacturing wave? The role of critical success factors. Technological Forecasting and Social Change, 132, 18-25. doi: 10.1016/j.techfore.2018.01.017

Dewangan, V., \& Godse, M. (2014). Towards a holistic enterprise innovation performance measurement system. Technovation, 34(9), 536-545. doi: 10.1016/ j.technovation.2014.04.002

Dimara, E., \& Skuras, D. (2003). Adoption of agricultural innovations as a two-stage partial observability process. Agricultural Economics, 28(3), 187-196. doi: 10.1111/j.1574-0862.2003.tb00137.x

Dodgson, M., \& Hinze, S. (2000). Indicators used to measure the innovation process: Defects and possible remedies. Research Evaluation, 9(2), 101-114. doi: $10.3152 / 147154400781777368$

Dziallas, M., \& Blind, K. (2018). Innovation indicators throughout the innovation process: An extensive literature analysis. Technovation, 80-81, 3-29. doi: 10.1016/j.technovation.2018.05.005

Edison, H., Bin Ali, N., \& Torkar, R. (2013). Towards innovation measurement in the software industry. Journal of Systems and Software, 86(5), 1390-1407. doi: 10.1016/j.jss.2013.01.013

Egorova, I. E., Gagarin, A. G., Kuznetsov, S. Y., Simonov, A. B., \& Velikanov, V. V. (2017). Successful Commercialization of Innovations as a Basis of Development of Modern Human Society. In Perspectives on the use of New Information and Communication Technology (ICT) in the Modern Economy (pp. 1156-1162). Cham, United Kingdom: Springer. doi: 10.1007/9783-319-90835-9_130

Espitia-Escuer, M., García-Cebrián, L. I., \& Muñoz-Porcar, A. (2014). Location as a competitive advantage for entrepreneurship an empirical application in the Region of Aragon (Spain). International Entrepreneurship and Management Journal, 11(1), 133-148. doi: 10.1007/s11365-014-0312-9

Evanschitzky, H., Eisend, M., Calantone, R. J., \& Jiang, Y. (2012). Success factors of product innovation: An updated meta-analysis. Journal of Product Innovation Management, 29, 21-37. doi: 10.1111/j.15405885.2012.00964.x
Ezzi, F., \& Jarboui, A. (2016). Does innovation strategy affect financial, social and environmental performance? Journal of Economics, Finance and Administrative Science, 21(40), 14-24. doi: 10.1016/j.jefas.2016.03.001

Frijns, B., Gilbert, A., Lehnert, T., \& Tourani-Rad, A. (2013). Uncertainty avoidance, risk tolerance and corporate takeover decisions. Journal of Banking \& Finance, 37(7), 2457-2471. doi: 10.1016/j.jbankfin.2013.02.010

Gallaud, D., \& Torre, A. (2005). Geographical proximity and the diffusion of knowledge. In Rethinking Regional Innovation and Change (pp. 127-146). New York, United States: Springer. doi: 10.1007/0-38723002-5_7

Garwood, S. G., Cox, L., Kaplan, V., Wasserman, N., \& Sulzer, J. L. (1980). Beauty is only "name" deep: the effect of first-name on ratings of physical attraction. Journal of Applied Social Psychology, 10(5), 431-435. doi:10.1111/j.1559-1816.1980.tb00721.x

Gault, F. (2018). Defining and measuring innovation in all sectors of the economy. Research Policy, 47(3), 617622. doi: 10.1016/j.respol.2018.01.007

Glass, R., Meissner, A., Gebauer, C., Stürmer, S., \& Metternich, J. (2018). Identifying the barriers to Industrie 4.0. Procedia CIRP, 72, 985-988. doi: $10.1016 / \mathrm{j}$. procir.2018.03.187

Gopalakrishnan, S., \& Damanpour, F. (1997). A review of innovation research in economics, sociology and technology management. Omega, International Journal of Management Science, 25(1), 15-28. doi: 10.1016/S0305-0483(96)00043-6

Gorecky, D., Schmitt, M., Loskyll, M., \& Zuhlke, D. (2014). Human-machine-interaction in the industry 4.0 era. 2014 12th IEEE International Conference on Industrial Informatics (INDIN). doi: 10.1109/indin.2014.6945523

Greenhalgh, T., Robert, G., Macfarlane, F., Bate, P., \& Kyriakidou, O. (2004). Diffusion of innovations in service organizations: systematic review and recommendations. The Milbank Quarterly, 82(4), 581-629. doi: 10.1111/j.0887-378X.2004.00325.x

Gülbahar, Y. (2007). Technology planning: A roadmap to successful technology integration in schools. Computers \& Education, 49, 943-956. doi: 10.1016/j. compedu.2005.12.002

Habicht, H., Möslein, K. M., \& Reichwald, R. (2012). Open innovation maturity. International Journal of Knowledge-Based Organizations, 2(1), 92-111. doi: 0.1142/ S1363919611003696

Ham, J., Lee, J. N., Kim, D. J., \& Choi, B. (2015). Open innovation maturity model for the government: An open system perspective. Proceedings of the $36^{\text {th }}$ International Conference on Information Systems, Fort Worth, Texas, United States.

Hameed, M. A., Counsell, S., \& Swift, S. (2012). A conceptual model for the process of IT innovation adoption in organizations. Journal of Engineering and Technology Management, 29(3), 358-390. doi: 10.1016/j.jengtecman.2012.03.007

Hart, S., Jan Hultink, E., Tzokas, N., \& Commandeur, H. R. (2003). Industrial companies' evaluation crite- 
ria in new product development gates. Journal of Product Innovation Management, 20(1), 22-36. doi: 10.1111/1540-5885.201003

Hassan, H. (2017). Organizational factors affecting cloud computing adoption in small and medium enterprises (SMEs) in service sector. International Conference on Enterprise Information Systems, Barcelona, Spain, 976-981. doi: 10.1016/j.procs.2017.11.126

Hermann, M., Pentek, T., \& Otto, B. (2016). Design principles for Industrie 4.0 scenarios. 2016 49th Hawaii International Conference on System Sciences (HICSS). doi: 10.1109/hicss.2016.488

Hinnant, C. C., \& O’Looney, J. A. (2003). Examining preadoption interest in online innovations: An exploratory study of e-service personalization in the public sector. IEEE Transactions on Engineering Management, 50(4), 436-447. doi: 0.1109/TEM.2003. 820133

Hoffman, D. G. (2002). Managing operational risk: 20 organization-wide best practice strategies. New York, United States: John Wiley \& Sons.

Hsu, C. L., \& Lin, J. C. C. (2016). Exploring factors affecting the adoption of internet of things services. Journal of Computer Information Systems, 58(1), 49-57. doi: 10.1080/08874417.2016.1186524

Issa, A., Hatiboglu, B., Bildstein, A., \& Bauernhansl, T. (2018). Industrie 4.0 roadmap: Framework for digital transformation based on the concepts of capability maturity and alignment. Procedia CIRP, 72, 973-978.

Issar, G., \& Navon, L. R. (2016). Operational Excellence. In G. Issar, \& L. R. Navon (Eds.), Manufacturing Overhead (MOH) and Departmental Expense Control (pp. 91-93). Springer International Publishing.

Jazdi, N. (2014). Cyber-physical systems in the context of Industry 4.0. 2014 IEEE International Conference on Automation, Quality and Testing, Robotics. doi: 10.1109/aqtr.2014.6857843

Jeyaraj, A., Rottman, J. W., \& Lacity, M. C. (2006). A review of the predictors, linkages, and biases in IT innovation adoption research. Journal of Information Technology, 21, 1-23. doi: 10.1057/palgrave.jit.2000056

Joachim, V., Spieth, P., \& Heidenreich, S. (2018). Active innovation resistance: An empirical study on functional and psychological barriers to innovation adoption in different contexts. Industrial Marketing Management, 71, 95-107. doi: 10.1016/j.indmarman.2017.12.011

Joia, L. A., Gutman, L. F., \& Moreno, V. (2016). Intention of use of home broker systems from the stock market investors' perspective. The Journal of High Technology Management Research, 27(2), 184-195. doi: 10.1016/j.hitech.2016.10.008

Kagermann, H., Helbig, J., Hellinger, A., \& Wahlster, W. (2013). Recommendations for implementing the strategic initiative INDUSTRIE 4.0: Securing the future of German manufacturing industry, final report of the Industrie 4.0 Working Group. Forschungs Union.

Kamble, S. S., Gunasekaran, A., \& Gawankar, S. A. (2018). Sustainable Industry 4.0 framework: A systematic literature review identifying the current trends and future perspectives. Process Safety and Environmental Protection, 117, 408-425. doi: 10.1016/j. psep.2018.05.009
Kang, H. S., Lee, J. Y., Choi, S. S., Kim, H., Park, J. H., Son, J. Y., Kim, B. H., \& Noh, S. D. (2016). Smart manufacturing: Past research, present findings, and future directions. International Journal of Precision Engineering and Manufacturing-Green Technology, 3(1), 111-128. doi: 10.1007/s40684-016-0015-5

Kerschner, C., \& Ehlers, M. (2016). A framework of attitudes towards technology in theory and practice. Ecological Economics, 126, 139-151. doi: 10.1016/j. ecolecon.2016.02.010

Kolberg, D., \& Zühlke, D. (2015). Lean automation enabled by industry 4.0 technologies. IFAC-PapersOnLine, 48(3), 1870-1875. doi: 10.1016/j.ifacol.2015.06.359

Lee, J., Bagheri, B., \& Kao, H. A. (2015). A cyber-physical systems architecture for Industry 4.0-based manufacturing systems. Manufacturing Letters, 3, 18-23. doi: 10.1016/j.mfglet.2014.12.001

Lee, J. H., Phaal, R., \& Lee, S.-H. (2013). An integrated service-device-technology roadmap for smart city development. Technological Forecasting and Social Change, 80(2), 286-306. doi: 10.1016/j.techfore.2012.09.020

Letia, T., \& Kilyen, A. (2018). Using unified enhanced time Petri net models for cyber-physical system development. International Federation of Automatic Control PapersOnLine, 51(2), 248-253. doi: 10.1016/j.ifacol.2018.03.043

Li, X., Ishii, H., \& Masuda, T. (2012). Single machine batch scheduling problem with fuzzy batch size. Computers \& Industrial Engineering, 62(3), 688-692. doi: 10.1016/j.cie.2011.12.021

Liao, Y., Deschamps, F., Loures, E. D. F. R., \& Ramos, L. F. P. (2017). Past, present and future of Industry 4.0-a systematic literature review and research agenda proposal. International Journal of Production Research, 55(12), 3609-3629. doi: 10.1080/00207543.2017.1308576

Lira, V., Tavares, E., \& Maciel, P. (2015). An automated approach to dependability evaluation of virtual networks. Computer Networks, 88(9), 89-102. doi: 10.1016/j.comnet.2015.05.016

Lombardi, P., Giordano, S., Farouh, H., \& Yousef, W. (2012). Modelling the smart city performance. Innovation: The European Journal of Social Science Research, 25(2), 137-149. doi: 10.1080/13511610.2012.660325

Lopez, J., \& Rubio, J. E. (2018). Access control for cyberphysical systems interconnected to the cloud. Computer Networks, 134, 46-54. doi: 10.1016/j.comnet.2018.01.037

Lu, Y. (2017). Industry 4.0: A survey on technologies, applications and open research issues. Journal of Industrial Information Integration, 6, 1-10. doi: 10.1016/j. jii.2017.04.005

Manral, L. (2010). Demand competition and investment heterogeneity in industries based on systemic technologies: Evidence from the US long-distance telecommunications services industry, 1984-1996. Journal of Evolutionary Economics, 20(5), 765-802. doi: 10.1007/s00191-010-0175-3

Martínez-Noya, A., \& García-Canal, E. (2017). Location, shared suppliers and the innovation per- 
formance of R\&D outsourcing agreements. Industry and Innovation, 25(3), 308-332. doi: 10.1080/13662716.2017.1329085

Mathiassen, L., \& Munk-Madsen, A. (2007). Formalizations in systems development. Behaviour and Information Technology, 5(2), 145-155. doi: 10.1080/01449298608914507

Mehrad, D., \& Mohammadi, S. (2017). Word of Mouth impact on the adoption of mobile banking in Iran. Telematics and Informatics, 34(7), 1351-1363. doi: https://doi.org/10.1016/j.tele.2016.08.009

Meyer, A. D., \& Goes, J. B. (1988). Organizational assimilation of innovations: A multilevel contextual analysis. Academy of Management Journal, 31(4), 897-923. doi: $10.5465 / 256344$

Miranda, M. Q., Farias, J. S., De Araújo Schwartz, C., \& De Almeida, J. P. (2016). Technology adoption in diffusion of innovations perspective: Introduction of an ERP system in a non-profit organization. RAI Revista de Administração e Inovação, 13(1), 48-57. doi: 10.1016/j.rai.2016.02.002

Miremadi, I., Saboohi, Y., \& Jacobsson, S. (2018). Assessing the performance of energy innovation systems: Towards an established set of indicators. Energy Research \& Social Science, 40, 159-176. doi: 10.1016/j. erss.2018.01.002

Molina, E., \& Jacob, E. (2018). Software-defined networking in cyber-physical systems: A survey. Computers \& Electrical Engineering, 66, 407-419. doi: 10.1016/j. compeleceng.2017.05.013

Monostori, L., Kádár, B., Bauernhansl, T., Kondoh, S., Kumara, S., Reinhart, G., Sauer, O., Schuh, G., Sihn, W., \& Ueda, K. (2016). Cyber-physical systems in manufacturing. CIRP Annals - Manufacturing Technology, 65(2), 621-641. doi: 10.1016/j.cirp.2016.06.005

Morrar, R., Arman, H., \& Mousa, S. (2017). The fourth industrial revolution (Industry 4.0): A social innovation perspective. Technology Innovation Management Review, 7(11), 12-20. doi: 10.22215/timreview/1117

Müller, J. M. (2019). Antecedents to digital platform usage in Industry 4.0 by established manufacturers. Sustainability, 11(4), 1121. doi: 10.3390/su11041121

Müller, J. M., Kiel, D., \& Voigt, K. (2018). What drives the implementation of Industry 4.0? The role of opportunities and challenges in the context of sustainability. Sustainability, 10(1), 247. doi:10.3390/su10010247

O’Hern, M. S., \& Rindfleisch, A. (2017). Customer co-creation: A typology and research agenda. In Review of marketing research (pp. 108-130). Routledge.

Oesterreich, T. D., \& Teuteberg, F. (2016). Understanding the implications of digitisation and automation in the context of Industry 4.0: A triangulation approach and elements of a research agenda for the construction industry. Computers in Industry, 83, 121-139. doi: 10.1016/j.compind.2016.09.006

Organization for Economic Cooperation and Development (OECD). (2005). Oslo Manual: The measurement of scientific and technological activities. Proposed Guidelines for Collecting an Interpreting Technological Innovation Data.

Oyemomi, O., Liu, S., Neaga, I., Chen, H., \& Nakpodia, F. (2019). How cultural impact on knowledge sharing contributes to organizational performance: Using the fsQCA approach. Journal of Business Research, 94, 313-319. doi: 10.1016/j. jbusres.2018.02.027

Pilke, E. (2004). Flow experiences in information technology use. International Journal of HumanComputer Studies, 61(3), 347-357. doi: 10.1016/j. ijhcs.2004.01.004

Plsek, P. (2003). Complexity and the adoption of innovation in health care. Accelerating quality improvement in health care: Strategies to accelerate the diffusion of evidence-based innovations. Washington, United States: National Institute for Healthcare Management Foundation and National Committee for Quality in Health Care.

Prest A. R., \& Turvey R. (1966) Cost-Benefit Analysis: A Survey. In Surveys of Economic Theory. London, United Kingdom: Palgrave Macmillan. doi: 10.1007/978-1-349-00210-8_5

Priyadarshinee, P., Raut, R. D., Jha, M. K., \& Kamble, S. S. (2017). A cloud computing adoption in Indian SMEs: Scale development and validation approach. Journal of High Technology Management Research, 28(2), 221-245. doi: 10.1016/j.hitech.2017.10.010

Rajnai, Z., \& Kocsis, I. (2018). Assessing industry 4.0 readiness of enterprises. 2018 IEEE 16th World Symposium on Applied Machine Intelligence and Informatics (SAMI), IEEE.

Rogers, E. M. (1995). Diffusion of Innovations. Fourth Ed. New York, United States: Free Press.

Rojko, A. (2017). Industry 4.0 concept: Background and overview. International Journal of Interactive Mobile Technologies, 11(5), 77-90. doi: 10.3991/ijim. v11i5.7072

Sabherwal, R., \& King, W. (1991). Towards a theory of strategic use of information resources: An inductive approach. Information and Management, 20, 191-212. doi: 10.1016/0378-7206(91)90055-7

Salleh, M., Bahari, M., \& Zakaria, N. H. (2017). An overview of software functionality service: A systematic literature review. Procedia Computer Science, 124, 337-344. doi: 10.1016/j.procs.2017.12.163

Schumpeter, J. A. (1934). Change and the Entrepreneur. Essays of JA Schumpeter, 4(23), 45-91.

Shamim, S., Cang, S., Yu, H., \& Li, Y. (2016). Management approaches for Industry 4.0: A human resource management perspective. 2016 IEEE Congress on Evolutionary Computation (CEC), IEEE.

Sharma, S. K., Al-Badi, A. H., Govindaluri, S. M., \& AlKharusi, M. H. (2016). Predicting motivators of cloud computing adoption: A developing country perspective. Computers in Human Behavior, 62, 6169. doi: 10.1016/j.chb.2016.03.073

Siderska, J., \& Mubarok, K. (2018). Cloud Manufacturing Platform and Architecture Design. Multidisciplinary Aspects of Production Engineering, 1(1), 673-680. doi: 10.2478/mape-2018-0085

Slater, S. F., \& Mohr, J. J. (2006). Successful development and commercialization of technological innovation: Insights based on strategy type. Journal of Product Innovation Management, 23(1), 26-33. doi: 10.1111/j.1540-5885.2005.00178.x 
Solis, B. (2016). The six stages of digital transformation maturity. Retrieved from https://www. prophet. com/2016/04/the--six--stages--of--digital--transformation.

Song, J. (2014). Understanding the adoption of mobile innovation in China. Computers in Human Behavior, 38, 339-348. doi: 10.1016/j.chb.2014.06.016

Sosna, M., Trevinyo-Rodriguez, R. N., \& Velamuri, S. R. (2010). Business model innovation through trialand-error learning: The Naturhouse case. Long Range Planning, 43(2), 383-407. doi: 10.1016/ j.lrp.2010.02.003

Stock, T., \& Seliger, G. (2016). Opportunities of sustainable manufacturing in industry 4.0. Procedia CIRP, 40, 536-541. doi: 10.1016/j.procir.2016.01.129

Straub, E. T. (2009). Understanding technology adoption: Theory and future directions for informal learning. Review of Educational Research, 79(2), 625-649. doi: $10.3102 / 0034654308325896$

Sung, T. K. (2018). Industry 4.0: A Korea perspective. Technological Forecasting \& Social Change, 132, 40-45. doi: 10.1016/j.techfore.2017.11.005

Suomala, P. (2004). The life cycle dimension of new product development performance measurement. International Journal of Innovation Management, 8(02), 193-221. doi: 10.1142/S1363919604001039

Szczerbicki, E. (2008). Smart Systems Integration: Toward overcoming the problem of complexity. Cybernetics and Systems, 39(2), 190-198. doi: 10.1080/01969720701853475

Tao, F., Qi, Q., Liu, A., \& Kusiak, A. (2018). Data-driven smart manufacturing. Journal of Manufacturing Systems, 48, 157-169. doi: 10.1016/j.jmsy.2018.01.006

Terziyan, V., Gryshko, S., \& Golovianko, M. (2018). Patented intelligence: Cloning human decision models for Industry 4.0. Journal of Manufacturing Systems, 48, 204-217. doi: 10.1016/j.jmsy.2018.04.019

Tweedale J. W. (2015). Enhancing the degree of autonomy by creating automated components within a multiagent system framework. In J. Tweedale, L. Jain, J. Watada, \& R. Howlett (Eds.), Knowledge-Based Information Systems in Practice. Smart Innovation, Systems and Technologies. Cham, United Kingdom: Springer. doi: 10.1007/978-3-319-13545-8_15

van Oorschot, J. A., Hofman, E., \& Halman, J. I. (2018). A bibliometric review of the innovation adoption literature. Technological Forecasting and Social Change, 134, 1-21. doi: 10.1016/j.techfore.2018.04.032

Vogel-Heuser, B., \& Hess, D. (2016). Guest editorial Industry 4.0-prerequisites and visions. IEEE Transactions on Automation Science and Engineering, 13(2), 411413. doi: 10.1109/TASE.2016.2523639

Wang, B., Zhao, J., Wan, Z., Ma, J., Li, H., \& Ma, J. (2016). Lean intelligent production system and value stream practice. 3rd International Conference on Economics and Management (ICEM 2016). doi:10.12783/dtem/ icem2016/4106

Wegner, A., Graham, J., \& Ribble, E. (2017). A new approach to cyberphysical security in industry 4.0. In Cybersecurity for Industry 4.0 (pp. 59-72). Cham, United Kingdom: Springer. doi: 10.1007/978-3-31950660-9_3
$\mathrm{Xu}, \mathrm{Z}$. (2006). A note on linguistic hybrid arithmetic averaging operator in multiple attribute group decision making with linguistic information. Group Decision and Negotiation, 15(6), 593-604. doi: 10.1007/ s10726-005-9008-4

Yigitcanlar, T., Sabatini-Marques, J., da-Costa, E. M., Kamruzzaman, M., \& Ioppolo, G. (2019). Stimulating technological innovation through incentives: Perceptions of Australian and Brazilian firms. Technological Forecasting and Social Change, 146, 403-412. doi: 10.1016/j.techfore.2017.05.039

Zhang, M., \& Hartley, J. L. (2018). Guanxi, IT systems, and innovation capability: the moderating role of proactiveness. Journal of Business Research, 90, 75-86. doi: 10.1016/j.jbusres.2018.04.036

Zhu, K., Dong, S., Xu, S. X., \& Kraemer, K. L. (2006). Innovation diffusion in global contexts: Determinants of post-adoption digital transformation of European companies. European Journal of Information Systems, 15(6), 601-616. doi: 10.1057/palgrave. ejis. 3000650 